\title{
Multidimensional Heisenberg convolutions and product formulas for multivariate Laguerre polynomials
}

\author{
Michael Voit \\ Fakultät Mathematik, Technische Universität Dortmund \\ Vogelpothsweg 87 \\ D-44221 Dortmund, Germany \\ e-mail: michael.voit@math.uni-dortmund.de
}

\begin{abstract}
Let $p, q$ positive integers. The groups $U_{p}(\underline{\mathrm{C}})$ and $U_{p}(\underline{\mathrm{C}}) \times U_{q}(\underline{\mathrm{C}})$ act on the Heisenberg group $H_{p, q}:=M_{p, q}(\underline{\mathrm{C}}) \times \underline{\mathrm{R}}$ canonically as groups of automorphisms where $M_{p, q}(\underline{\mathrm{C}})$ is the vector space of all complex $p \times q$ matrices. The associated orbit spaces may be identified with $\Pi_{q} \times \underline{\mathrm{R}}$ and $\Xi_{q} \times \underline{\mathrm{R}}$ respectively with the cone $\Pi_{q}$ of positive semidefinite matrices and the Weyl chamber $\Xi_{q}=\left\{x \in \underline{\mathrm{R}}^{q}: x_{1} \geq \ldots \geq x_{q} \geq 0\right\}$.

In this paper we compute the associated convolutions on $\Pi_{q} \times \underline{\mathrm{R}}$ and $\Xi_{q} \times \underline{\mathrm{R}}$ explicitly depending on $p$. Moreover, we extend these convolutions by analytic continuation to series of convolution structures for arbitrary parameters $p \geq 2 q-1$. This leads for $q \geq 2$ to continuous series of noncommutative hypergroups on $\Pi_{q} \times \underline{\mathrm{R}}$ and commutative hypergroups on $\Xi_{q} \times \underline{\mathrm{R}}$. In the latter case, we describe the dual space in terms of multivariate Laguerre and Bessel functions on $\Pi_{q}$ and $\Xi_{q}$. In particular, we give a non-positive product formula for these Laguerre functions on $\Xi_{q}$.

The paper extends the known case $q=1$ due to Koornwinder, Trimeche, and others as well as the group case with integers $p$ due to Faraut, Benson, Jenkins, Ratcliff, and others. Moreover, it is closely related to product formulas for multivariate Bessel and other hypergeometric functions of Rösler.
\end{abstract}

2010 Mathematics Subject Classification: Primary 43A62; Secondary 33C67. 33C52, 43A $90,43 \mathrm{~A} 20$

Key words and phrases: Heisenberg convolution, matrix cones, Weyl chambers, multivariate Laguerre polynomials, multivariate Bessel functions, product formulas, hypergroups, hypergroup characters. 


\section{Introduction}

For positive integers $p \geq q$ consider the vector space $M_{p, q}$ of all $p \times q$ matrices over $\underline{\mathrm{C}}$. Consider the associated Heisenberg group $H_{p, q}:=M_{p, q} \times \underline{\mathrm{R}}$ with the product

$$
(x, a) \cdot(y, b)=\left(x+y, a+b-\operatorname{Im} \operatorname{tr}\left(x^{*} y\right)\right)
$$

where $\operatorname{tr}$ denotes the trace of the $q \times q$ matrix $x^{*} y$. Clearly, the unitary groups $K:=U_{p}:=U_{p}(\underline{\mathrm{C}})$ and $K:=U_{p} \times U_{q}$ act on $H_{p, q}$ via

$$
u(x, a):=(u x, a) \quad \text { and } \quad(u, v)(x, a):=\left(u x v^{*}, a\right)
$$

for $u \in U_{p}, v \in U_{q}, x \in M_{p, q}$, and $a \in \underline{\mathrm{R}}$ respectively as groups of automorphisms. The associated orbit spaces may be identified with $\Pi_{q} \times \underline{\mathrm{R}}$ and $\Xi_{q} \times \underline{\mathrm{R}}$ respectively with the cone $\Pi_{q}$ of complex positive semidefinite matrices and the Weyl chamber $\Xi_{q}=\left\{x \in \underline{\mathrm{R}}^{q}: x_{1} \geq \ldots \geq x_{q} \geq 0\right\}$ of type $B$. It is well-known that the Banach-*- algebras $M_{b}\left(H_{p, q}, K\right)$ of all $K$-invariant bounded regular Borel measures with the convolution as multiplication are commutative always for $K:=U_{p} \times U_{q}$, and for $K:=U_{p}$ for $q=1$ only (in which case the cases $K:=U_{p}:=U_{p}(\underline{\mathrm{C}})$ and $K:=U_{p} \times U_{1}$ lead to the same result). Moreover, in these Gelfand pair cases, the associated spherical functions are well-known in terms of multivariate Laguerre and Bessel functions; we refer to [BJR1], BJR2], [BJRW], [C], [F], [Kac, [W] and references there for this topic.

In this paper we compute the associated orbit convolutions on $\Pi_{q} \times \underline{\mathrm{R}}$ and $\Xi_{q} \times \underline{\mathrm{R}}$ explicitly depending on the dimension parameter $p$. This computation is similar to that of Rösler [R2] where the action of $U_{p}$ and $U_{p} \times U_{q}$ on $M_{p, q}$ is considered for the fields $\underline{\mathrm{R}}, \underline{\mathrm{C}}, \underline{\mathrm{H}}$, and where multivariate Bessel functions appear as spherical functions. Moreover, following [R2], we extend these convolutions by analytic continuation to series of convolution structures for arbitrary parameters $p \geq 2 q-1$ by using the famous theorem of Carleson. This extension leads for $q \geq 2$ to continuous series of noncommutative hypergroups on $\Pi_{q} \times \underline{\mathrm{R}}$ and continuous series of commutative hypergroups on $\Xi_{q} \times \underline{\mathrm{R}}$. In the latter case, we shall describe the dual spaces in terms of multivariate Laguerre and Bessel functions on $\Pi_{q}$ and $\Xi_{q}$. Moreover, we determine further data of these hypergroups like the Haar measures, the Plancherel measures, automorphisms and subhypergroups.

The main results will be as follows: For the (for $q \geq 2$ noncommutative) hypergroup structures on $\Pi_{q} \times \underline{\mathrm{R}}$ we shall derive in Section 2 :

1.1 Theorem. Let $q \geq 1$ be an integer and $p \in] 2 q-1, \infty[$. Define a convolution of point measures on $\Pi_{q} \times \underline{R}$ by

$$
\begin{aligned}
& \left(\delta_{(r, a)} *_{p, q} \delta_{(s, b)}\right)(f)= \\
& =\kappa_{p, q} \int_{B_{q}} f\left(\sqrt{r^{2}+s^{2}+r w s+(r w s)^{*}}, a+b-\operatorname{Im} \operatorname{tr}(r w s)\right) \\
& \cdot \Delta\left(I_{q}-w^{*} w\right)^{p-2 q} d w
\end{aligned}
$$


for $f \in C_{b}\left(\Pi_{q} \times \underline{R}\right), r, s \in \Pi_{q}, a, b \in \underline{R}$, where

$$
B_{q}:=\left\{w \in M_{q, q}: w^{*} w<I_{q} \text {, i.e., } I_{q}-w^{*} w \text { is positive definite }\right\},
$$

$\Delta$ is the determinant of a $q \times q$ matrix, and $\kappa_{p, q}>0$ is a suitable constant. Then this formula establishes by unique bilinear, weakly continuous extension an associative convolution on $M_{b}\left(\Pi_{q} \times \underline{R}\right)$. More precisely, $\left(\Pi_{q} \times \underline{R}, *_{p, q}\right)$ is a hypergroup in the sense of Jewett (see $[B H],[J])$ with $(0,0)$ as identity and with the involution $\overline{(r, a)}:=(r,-a)$. Moreover,

$$
\omega_{p, q}(f)=\int_{\Pi_{q} \times \underline{R}} f(\sqrt{r}, a) \Delta(r)^{p-q} d r d a
$$

defines a left and right Haar measure.

For the commutative hypergroup structures on $\Xi_{q} \times \underline{\mathrm{R}}$ we shall derive in Section 3:

1.2 Theorem. Let $q \geq 1$ be an integer and $p \in] 2 q-1, \infty\left[\right.$. Then $\Xi_{q} \times \underline{R}$ carries a commutative hypergroup structure with convolution

$$
\begin{aligned}
& \left(\delta_{(\xi, a)} \circ_{p, q} \delta_{(\eta, b)}\right)(f) \\
& =\kappa_{p, q} \int_{B_{q}} \int_{U_{q}} f\left(\sigma\left(\sqrt{\xi^{2}+u \eta^{2} u^{*}+\xi w u \eta u^{*}+u \eta u^{*} w^{*} \xi}\right),\right. \\
& \left.a+b-\operatorname{Im} \operatorname{tr}\left(\xi w u \eta u^{*}\right)\right) \cdot \Delta\left(I_{q}-w^{*} w\right)^{p-2 q} d u d w
\end{aligned}
$$

for $f \in C_{b}\left(\Xi_{q} \times \underline{R}\right),(\xi, a),(\eta, b) \in \Xi_{q} \times \underline{R}$ where du means integration with respect to the normalized Haar measure on $U_{q}$ and $\xi \in \Xi$ is identified with the associated diagonal matrix in $\Pi_{q}$. The neutral element of this hypergroup is given by $(0,0) \in \Xi_{q} \times \underline{R}$, and the involution by $\overline{(\xi, a)}:=(\xi,-a)$. Moreover, a Haar measure is given by $d \tilde{\omega}_{p, q}(\xi, a):=h_{p, q}(\xi) d \xi$ da with the Lebesgue density

$$
h_{p, q}(\xi):=\prod_{i=1}^{q} \xi_{i}^{2 p-2 q+1} \prod_{i<j}\left(\xi_{i}^{2}-\xi_{j}^{2}\right)^{2} .
$$

Moreover, the dual spaces of these commutative hypergroups, i.e., the sets of all bounded continuous multiplicative functions, will be described precisely as a Heisenberg fan consisting of multivariate Laguerre and Bessel functions which were studied in $[\mathrm{BF}],[\mathrm{F}],[\mathrm{FK}],[\mathrm{He}]$ and many others. As already noticed above, this description is well known for the group cases with integer $p$ by [BJRW], [F].

In Section 4 we shall use the product formula on $\Xi_{q} \times \underline{\mathrm{R}}$ of Section 3 in order to derive a product formula for the normalized Laguerre functions

$$
\tilde{\varphi}_{\mathbf{m}}^{p}(x):=\frac{l_{\mathbf{m}}^{p}\left(x^{2} / 2\right)}{l_{\mathbf{m}}^{p}(0)}=e^{-\left(x_{1}^{2}+\ldots+x_{q}^{2}\right) / 2} \frac{L_{\mathbf{m}}^{p}\left(x^{2}\right)}{L_{\mathbf{m}}^{p}(0)} \quad\left(x \in \Xi_{q}\right)
$$


for $p>2 q-1$ which are introduced, for instance, in [FK]. We shall show that for all partitions $\mathbf{m}$ and all $\xi, \eta \in \Xi_{q}$,

$$
\begin{array}{r}
\tilde{\varphi}_{\mathbf{m}}^{p}(\xi) \cdot \tilde{\varphi}_{\mathbf{m}}^{p}(\eta)=\kappa_{p, q} \int_{B_{q}} \int_{U_{q}} \tilde{\varphi}_{\mathbf{m}}^{p}\left(\sigma\left(\sqrt{\xi^{2}+u \eta^{2} u^{*}+\xi w u \eta u^{*}+u \eta u^{*} w^{*} \xi}\right)\right) \\
\cdot e^{-i \cdot \operatorname{Im} \operatorname{tr}\left(\xi w u \eta u^{*}\right)} \Delta\left(I_{q}-w^{*} w\right)^{p-2 q} d u d w .
\end{array}
$$

For $q=1$, this formula was derived by Koornwinder [Ko] who also gave another version of this product formula using Bessel functions.

We here notice that on all three levels discussed above also degenerated product formulas are available for the limit case $p=2 q-1$. We do not consider the case $p<2 q-1$.

It is a pleasure to thank Margit Rösler for many valuable discussions about their multivariate Bessel convolutions and multivariate special functions.

\section{Heisenberg convolutions associated with ma- trix cones}

For positive integers $p, q$ consider the vector space $M_{p, q}$ of all $p \times q$ matrices over $\underline{\mathrm{C}}$. Consider the associated Heisenberg group $H_{p, q}:=M_{p, q} \times \underline{\mathrm{R}}$ with the product

$$
(x, a) \cdot(y, b)=\left(x+y, a+b-\operatorname{Im} \operatorname{tr}\left(x^{*} y\right)\right)
$$

where $\operatorname{tr}$ denotes the trace of the $q \times q$ matrix $x^{*} y$. Clearly, the unitary group $U_{p}:=U_{p}(\underline{\mathrm{C}})$ acts on $H_{p, q}$ via

$$
u(x, a):=(u x, a) \quad \text { for } \quad u \in U_{p}, x \in M_{p, q}, a \in \underline{\mathrm{R}}
$$

as a group of automorphisms. We regard $U_{p}$ as a compact subgroup of the associated semidirect product $G_{p, q}:=U_{p} \ltimes H_{p, q}$ in the natural way and consider the double coset space $G_{p, q} / / U_{p}$ which may be also regarded as the space of all orbits of the action of $U_{p}$ on $H_{p, q}$ in the canonical way. Moreover, using uniqueness of polar decomposition of $p \times q$ matrices, we see immediately that we may identify this space of orbits also with the space $\Pi_{q} \times \underline{\mathrm{R}}$ with

$$
\Pi_{q}:=\left\{z \in M_{q, q}: z \text { Hermitian and positive semidefinite }\right\}
$$

via

$$
U_{p}((x, a)) \simeq(|x|, a),
$$

where $|x|:=\sqrt{x^{*} x} \in \Pi_{q}$ stands for the unique positive semidefinite square root of $x^{*} x \in \Pi_{q}$.

Now consider the Banach-*-algebra $M_{b}\left(G_{p, q} \| U_{p}\right)$ of all $U_{p}$-biinvariant bounded signed Borel measures on $G_{p, q}$ with the usual convolution of measures as multiplication. If we extend the canonical projection $P: G_{p, q} \rightarrow$ 
$G_{p, q} / / U_{p} \simeq \Pi_{q} \times \underline{\mathrm{R}}$ to measures by taking images of measures w.r.t. $P$, this extension becomes an isometric isomorphism between the Banach spaces $M_{b}\left(G_{p, q}|| U_{p}\right)$ and $M_{b}\left(\Pi_{q} \times \underline{\mathrm{R}}\right)$. We thus may transfer the Banach-*-algebra structure on $M_{b}\left(G_{p, q}|| U_{p}\right)$ to $M_{b}\left(\Pi_{q} \times \underline{\mathrm{R}}\right)$ by this isomorphism and obtain a probability preserving, weakly continuous convolution $*_{p, q}$ on $M_{b}\left(\Pi_{q} \times \underline{\mathrm{R}}\right)$ in this way. The pair $\left(\Pi_{q} \times \underline{\mathrm{R}}, *_{p, q}\right)$ forms a so-called hypergroup; for general details on hypergroups the the construction above via double cosets and orbits we refer to $[\mathrm{BH}]$ and $[\mathrm{J}]$.

Clearly, this Heisenberg-type convolution $*_{p, q}$ on (measures on) $\Pi_{q} \times$ $\underline{\mathrm{R}}$ is commutative iff so is $M_{b}\left(G_{p, q} \| U_{p}\right)$, i.e., iff $\left(G_{p, q}, U_{p}\right)$ is a Gelfand pair. As Gelfand pairs associated with Heisenberg groups were classified completely (see [BJR2], [C], [Kac], [W]), it turns out that the convolution $*_{p, q}$ is commutative precisely for $q=1$. Moreover, for $q=1$, the convolutions $*_{p, q}$ on $\Pi_{1} \times \underline{\mathrm{R}}=[0, \infty[\times \underline{\mathrm{R}}$ and the associated hypergroup structures were investigated by several authors; see $[\mathrm{Ko}$, and the monographs $[\mathrm{T}],[\mathrm{BH}]$ as well as references therein.

We next compute the convolution $*_{p, q}$ for arbitrary positive integers $q$ under the technical restriction $p \geq 2 q$ which will become clear below. We do this by using the approach for the Gelfand pair $\left(U_{p} \ltimes M_{p, q}, U_{p}\right)$ in [R2] where the double coset space $\left(U_{p} \ltimes M_{p, q} / / U_{p}\right)$ is identified with $\Pi_{q}$, and where the same restriction appears. The computation here is only slightly more involved, and we obtain:

2.1 Proposition. Let $p \geq 2 q \geq 1$ be integers. Then the convolution $*_{p, q}$ of point measures is given by

$$
\begin{aligned}
& \left(\delta_{(r, a)} *_{p, q} \delta_{(s, b)}\right)(f) \\
& =\kappa_{p, q} \int_{B_{q}} f\left(\sqrt{r^{2}+s^{2}+r w s+(r w s)^{*}}, a+b-\operatorname{Im} \operatorname{tr}(r w s)\right) \\
& \cdot \Delta\left(I_{q}-w^{*} w\right)^{p-2 q} d w
\end{aligned}
$$

for $f \in C_{b}\left(\Pi_{q} \times \underline{R}\right), r, s \in \Pi_{q}, a, b \in \underline{R}$ with $I_{q} \in M_{q, q}$ the identity matrix,

$B_{q}:=\left\{w \in M_{q, q}: w^{*} w<I_{q}\right.$, i.e., $I_{q}-w^{*} w$ is positive definite $\}$,

$d w$ denoting integration w.r.t. Lebesgue measure on $M_{q, q}$,

$\Delta$ denoting the determinant of a $q \times q$ matrix, and

$$
\kappa_{p, q}:=\left(\int_{B_{q}} \Delta\left(I_{q}-w^{*} w\right)^{p-2 q} d w\right)^{-1}>0 .
$$

Proof. The canonical projection $\varphi: H_{p, q} \rightarrow H_{p, q}^{U_{p}} \simeq \Pi_{q} \times \underline{\mathrm{R}}$ from the Heisenberg group onto its orbit space is given explicitly by $\varphi(x, a)=(|x|, a)$ with $|x|:=\sqrt{x^{*} x}$. Moreover, if we define the block matrix

$$
\sigma_{0}:=\left(\begin{array}{c}
I_{q} \\
0
\end{array}\right) \in M_{p, q},
$$


an "orbit" $(r, a) \in \Pi_{q} \times \underline{\mathrm{R}}$ has the representative $\left(\sigma_{0} r, a\right) \in H_{p, q}$. By the general definition of the orbit convolution $*_{p, q}$ (see Section 8.2 of [J] or [R2]) we have

$$
\begin{aligned}
\left(\delta_{(r, a)} *_{p, q} \delta_{(s, b)}\right)(f) & =\left(\delta_{U_{p}\left(\sigma_{0} r, a\right)} *_{p, q} \delta_{U_{p}\left(\sigma_{0} s, b\right)}\right)(f) \\
& =\int_{U_{p}} f\left(\varphi\left(\left(\sigma_{0} r, a\right) \cdot u\left(\left(\sigma_{0} s, b\right)\right)\right) d u\right. \\
& =\int_{U_{p}} f\left(\left|\sigma_{0} r+u \sigma_{0} s\right|, a+b-\operatorname{Im} \operatorname{tr}\left(r \sigma_{0}^{*} u \sigma_{0} s\right)\right) d u
\end{aligned}
$$

where $d u$ denotes integration w.r.t. the normalized Haar measure on $U_{p}$. Using the definition of the absolute value of a matrix above and denoting the upper $q \times q$ block of $u$ by $u_{q}:=\sigma_{0}^{*} u \sigma_{0} \in M_{q, q}$, we readily obtain

$\left(\delta_{(r, a)}{ }_{p, q} \delta_{(s, b)}\right)(f)=\int_{U_{p}} f\left(\sqrt{r^{2}+s^{2}+r u_{q} s+\left(r u_{q} s\right)^{*}}, a+b-\operatorname{Im} \operatorname{tr}\left(r u_{q} s\right)\right) d u$.

The truncation lemma 2.1 of [R3] now implies the proposition.

2.2 Remarks. (1) The integral in Eq. (2.1) exists precisely for exponents $p-2 q>-1$ which shows that a formula for $*_{p, q}$ of the above kind is available precisely for $p \geq 2 q$.

(2) Let $p \geq 2 q \geq 1$ be integers, and let $f \in C_{b}\left(\Pi_{q} \times \underline{\mathrm{R}}\right), r, s \in \Pi_{q}, a, b \in \underline{\mathrm{R}}$. Formula (2.1) and a straightforward computation yield that

$$
\begin{aligned}
& \left(\delta_{(s, b)} *_{p, q} \delta_{(r, a)}\right)(f) \\
& =\kappa_{p, q} \int_{B_{q}} f\left(\sqrt{r^{2}+s^{2}+r w s+(r w s)^{*}}, a+b+\operatorname{Im} \operatorname{tr}(r w s)\right) \\
& \quad \cdot \Delta\left(I_{q}-w^{*} w\right)^{p-2 q} d w .
\end{aligned}
$$

If one compares this with Eq. (2.1), the reader can check directly the known fact that $*_{p, q}$ is non-commutative precisely for $q \geq 2$. For this, take for instance, $a=b=0, r=\left(\begin{array}{ll}1 & 0 \\ 0 & \mathbf{0}\end{array}\right)$, and $s=\left(\begin{array}{ll}\mathbf{0} & 0 \\ 0 & 1\end{array}\right)$ with the zero matrix $\mathbf{0} \in M_{q-1, q-1}$.

We next extend the definition of the Heisenberg convolution in Eq. (2.1) to noninteger exponents $p \in] 2 q-1, \infty[$ for a fixed dimension parameter $q$ by Carlson's theorem on analytic continuation. For the convenience of the reader we recapitulate this result from [Ti], p.186:

2.3 Theorem. Let $f(z)$ be holomorphic in a neighbourhood of $\{z \in \underline{C}$ : Re $z \geq 0\}$ satisfying $f(z)=O\left(e^{c|z|}\right)$ on Re $z \geq 0$ for some $c<\pi$. If $f(z)=$ 0 for all nonnegative integers $z$, then $f$ is identically zero for $\operatorname{Re} z>0$.

This theorem will lead to the following extended convolution: 
2.4 Theorem. Let $q \geq 1$ be an integer and $p \in] 2 q-1, \infty[$. Define the convolution of point measures on $\Pi_{q} \times \underline{R}$ by

$$
\begin{aligned}
& \left(\delta_{(r, a)} *_{p, q} \delta_{(s, b)}\right)(f) \\
& =\kappa_{p, q} \int_{B_{q}} f\left(\sqrt{r^{2}+s^{2}+r w s+(r w s)^{*}}, a+b-\operatorname{Im} \operatorname{tr}(r w s)\right) \\
& \quad \cdot \Delta\left(I_{q}-w^{*} w\right)^{p-2 q} d w
\end{aligned}
$$

for $f \in C_{b}\left(\Pi_{q} \times \underline{R}\right), r, s \in \Pi_{q}, a, b \in \underline{R}$ where $\kappa_{p, q}$, dw, $\Delta$ and other data are defined as in Proposition 2.1 above. Then this formula defines a weakly continuous convolution of point measures on $\Pi_{q} \times \underline{R}$ which can be extended uniquely in a bilinear, weakly continuous way to a probability preserving, weakly continuous, and associative convolution on $M_{b}\left(\Pi_{q} \times \underline{R}\right)$. More precisely, $\left(\Pi_{q} \times \underline{R}, *_{p, q}\right)$ is a hypergroup with $(0,0)$ as identity and with the involution $\overline{(r, a)}:=(r,-a)$.

Proof. It is clear from Eq. (2.3) that the mapping

$$
\left(\Pi_{q} \times \underline{\mathrm{R}}\right) \times\left(\Pi_{q} \times \underline{\mathrm{R}}\right) \rightarrow M_{b}\left(\Pi_{q} \times \underline{\mathrm{R}}\right), \quad((r, a),(s, b)) \mapsto \delta_{(r, a)} *_{p, q} \delta_{(s, b)}
$$

is probability preserving and weakly continuous. It is now standard (see [J]) to extend this convolution uniquely in a bilinear and weakly continuous way to a probability preserving convolution on $M_{b}\left(\Pi_{q} \times \underline{\mathrm{R}}\right)$.

To prove associativity, it suffices to consider point measures. So let $r, s, t \in \Pi_{q}, a, b, c \in \underline{\mathrm{R}}$, and $f \in C_{b}\left(\Pi_{q}\right)$. Then

$$
\begin{aligned}
& \delta_{(r, a)} *_{p, q}\left(\delta_{(s, b)} *_{p, q} \delta_{(t, c)}\right)(f) \\
& =\kappa_{p, q}^{2} \int_{B_{q}} \int_{B_{q}} f(H(r, a, s, b, t, c ; v, w)) \\
& \cdot \Delta\left(I_{q}-v^{*} v\right)^{p-2 q} \Delta\left(I_{q}-w^{*} w\right)^{p-2 q} d v d w=: I(p)
\end{aligned}
$$

with a certain argument $H$ independent of $p$. Similar,

$$
\left(\delta_{(r, a)} *_{p, q} \delta_{(s, b)}\right) *_{p, q} \delta_{(t, c)}(f)=: I^{\prime}(p)
$$

admits a similar integral representation with some integrand $H^{\prime}$ independent of $p$. The integrals $I(p)$ and $I^{\prime}(p)$ are well-defined and holomorphic in $\{p \in$ $\underline{\mathrm{C}}: \operatorname{Re} p>2 q-1\}$. Furthermore, we know from the group cases above that $I(p)=I^{\prime}(p)$ for all integers $p \geq 2 q$. As

$$
\left|\kappa_{p, q}\right|=O\left(|p|^{q^{2}}\right) \text { uniformly in }\{p \in \underline{\mathrm{C}}: \operatorname{Re} p>2 q-1\} \text { for } p \rightarrow \infty
$$

(see, for example, Eq. (3.9) of [R2]), we obtain readily that

$$
f(p):=I(p+2 q-1)-I^{\prime}(p+2 q-1)=O\left(|p|^{2 q^{2}}\right),
$$

and Theorem 2.3 ensures that $I(p)=I^{\prime}(p)$ for all $p>2 q-1$. Thus $*_{p, q}$ is associative. 
Finally, it is clear by Eq. (2.3) that $\delta_{(0,0)}$ is the neutral element. Moreover, as the support $\operatorname{supp}\left(\delta_{(r, a)} *_{p, q} \delta_{(s, b)}\right)$ of our convolution is obviously independent of $p \in] 2 q-1, \infty$ [, all further hypergroup axioms from [BH] or [J] regarding the support of convolution products are obvious, as they are valid for the group cases with integer $p \geq 2 q$.

2.5 Remark. The convolution (2.3) obviously satisfies the following support formula: For all $(r, a),(s, b) \in \Pi_{q} \times \underline{\mathrm{R}}$,

$$
\begin{aligned}
\operatorname{supp}\left(\delta_{(r, a)} *_{p, q} \delta_{(s, b)}\right) & \subset \\
& \subset\left\{(t, c) \in \Pi_{q} \times \underline{\mathrm{R}}:\|t\| \leq\|r\|+\|s\|,|c| \leq|a|+|b|+\|r\| \cdot\|s\|\right\}
\end{aligned}
$$

with the Euclidean norm $\|x\|:=\sqrt{\operatorname{tr}\left(x^{*} x\right)}$.

We next collect some properties of the hypergroups $\left(\Pi_{q} \times \underline{\mathrm{R}}, *_{p, q}\right)$ for $p \in] 2 q-1, \infty[$. We first turn to examples of automorphisms. For this we first recapitulate that a homeomorphism $T$ on $\Pi_{q} \times \underline{\mathrm{R}}$ is called a hypergroup automorphism, if for all $(r, a),(s, b) \in \Pi_{q} \times \underline{\mathrm{R}}$,

$$
T\left(\delta_{(r, a)} * \delta_{\overline{(s, b)}}\right)=\delta_{T(r, a)} * \delta_{\overline{T(s, b)}}
$$

where the left hand side means the image of the measure under $T$.

2.6 Lemma. For all $u \in U_{q}$ and $\lambda>0$, the mappings

$$
T_{u, \lambda}(r, a):=\left(\lambda u r u^{*}, \lambda^{2} a\right)
$$

are hypergroup automorphisms on $\left(\Pi_{q} \times \underline{R}, *_{p, q}\right)$.

Proof. Eq. (2.3) yields

$$
\begin{aligned}
& \left(\delta_{T_{u, \lambda}(r, a)^{*}}{ }_{p, q} \delta_{T_{u, \lambda}(s, b)}\right)(f) \\
& =\kappa_{p, q} \int_{B_{q}} f\left(\lambda \sqrt{u\left(r^{2}+s^{2}+r u^{*} w u s+\left(r u^{*} w u s\right)^{*}\right) u^{*}},\right. \\
& \lambda^{2}\left(a+b-\operatorname{Im} \operatorname{tr}\left(u_{\left.r u^{*} w u s u^{*}\right)}\right) \cdot \Delta\left(I_{q}-w^{*} w\right)^{p-2 q} d w .\right.
\end{aligned}
$$

Using $\operatorname{tr}(u t)=\operatorname{tr}(t u), \sqrt{u t u^{*}}=u \sqrt{t} u^{*}$ and the substitution $v=u^{*} w u$, we see that this expression is equal to

$$
\begin{aligned}
& \kappa_{p, q} \int_{B_{q}} f\left(\lambda u \sqrt{\left.r^{2}+s^{2}+r v s+(r v s)^{*}\right)} u^{*}\right. \\
& \lambda^{2}(a+b-\operatorname{Im} \operatorname{tr}(r v s)) \cdot \Delta\left(I_{q}-w^{*} w\right)^{p-2 q} d w \\
& =T_{u, \lambda}\left(\delta_{(r, a)} *_{p, q} \delta_{(s, b)}\right)
\end{aligned}
$$

as claimed. 
2.7 Remark. The Bessel hypergroups on the matrix cones $\Pi_{q}$ of [R2] admit many more hypergroup automorphisms. In fact, a complete classification of all automorphisms on these Bessel hypergroups is given in [V3]. Due to the additional term $\operatorname{Im} \operatorname{tr}(r w s)$ in Eq. (2.3), most of these hypergroup automorphisms on $\Pi_{q}$ cannot be extended to our Heisenberg convolutions.

We next turn to the (left) Haar measure which is unique up to a multiplicative constant by [J]:

2.8 Proposition. A left Haar measure of the hypergroup $\left(\Pi_{q} \times \underline{R}, *_{p, q}\right)$ is given by

$$
\omega_{p, q}(f)=\int_{\Pi_{q} \times \underline{R}} f(\sqrt{r}, a) \Delta(r)^{p-q} d r d a
$$

for a continuous function $f \in C_{c}\left(\Pi_{q} \times \underline{R}\right)$ with compact support and the restriction of the Lebesgue measure $d r$ on the vector space of all Hermitian $q \times q$ matrices.

Moreover, this left Haar measure is also a right Haar measure.

Proof. We first recall that the Heisenberg groups $H_{p, q}$ are unimodular with the usual Lebesgue measure $d \lambda$ as Haar measure. Therefore, by general results on orbit hypergroups (see e.g. [J]), the image $\varphi(d \lambda)$ of $d \lambda$ under the canonical projection $\varphi: H_{p, p} \rightarrow \Pi_{q} \times \underline{\mathrm{R}}$ is a left and right Haar measure on the hypergroup $\left(\Pi_{q} \times \underline{\mathrm{R}}, *_{p, q}\right)$. Moreover, the computation in Section 3.1 of [R2] shows that

$$
\varphi(d \lambda)(r, a)=c_{p, q} \cdot \Delta(r)^{p-q} d r d a \in M^{+}\left(\Pi_{q} \times \underline{\mathrm{R}}\right)
$$

with a certain known constant $c_{p, q}>0$. This proves the result for integers $p \geq 2 q$.

For the general case we must check that

$$
\begin{aligned}
& \int_{\Pi_{q}} \int_{\underline{\mathbf{R}}}\left(\delta_{(r, a)} *_{p, q} \delta_{(\sqrt{s}, b)}\right)(f) \Delta(s)^{p-q} d s d b \\
= & \int_{\Pi_{q}} \int_{\underline{\mathbf{R}}}\left(\delta_{(\sqrt{s}, b)} *_{p, q} \delta_{(r, a)}\right)(f) \Delta(s)^{p-q} d s d b \\
= & \int_{\Pi_{q}} \int_{\underline{\mathbf{R}}} f(\sqrt{s}, b) \Delta(s)^{p-q} d s d b
\end{aligned}
$$

for all $f \in C_{c}\left(\Pi_{q} \times \underline{\mathrm{R}}\right), r \in \Pi_{q}, a \in \underline{\mathrm{R}}$ and $p \in \underline{\mathrm{C}}$ with $\operatorname{Re} p>2 q-1$, where we use Eq. (2.3) also for the convolution for complex $p$. Clearly, all expressions are analytic in $p$ for fixed $f, r, a, q$. Moreover, by Eq. (2.3), all three expressions are bounded by

$$
C\|f\|_{\infty} \kappa_{p, q} \cdot M^{\operatorname{Re}(p-q)}
$$

with some constant $C$ and

$$
\begin{aligned}
M & :=\sup \left\{\Delta(s):(s, b) \in \Pi_{q} \times \underline{\mathrm{R}}, \operatorname{supp}\left(\delta_{(r, a)} *_{p, q} \delta_{(\sqrt{s}, b)}\right) \cap \operatorname{supp} f \neq \emptyset\right\} \\
& =\sup \left\{\Delta(s):(s, b) \in(r,-a) *_{p, q} \operatorname{supp}(f)\right\} .
\end{aligned}
$$


Using the estimate (2.4) for $\kappa_{p, q}$ and the estimate for the support of convolution products in Remark 2.5, we obtain that the necessary estimate in Carlson's theorem 2.3 holds whenever $\|r\|$ and the support of $f$ are contained in a sufficiently small neighborhood of $(0,0)$. Therefore, (2.6) holds in this case.

Finally, if $f \in C_{c}\left(\Pi_{q} \times \underline{\mathrm{R}}\right)$ and $r \in \Pi_{q}$ are arbitrary, then we choose a sufficiently small scaling parameter $\lambda$ such that $\lambda r$ and the support of $f_{\lambda}(s, a):=f\left(\lambda^{-1} s, \lambda^{-2} a\right)$ are sufficiently small such that (2.6) holds for $\lambda r$ and $f_{\lambda}$. As the scaling map $T_{I_{q}, \lambda}$ is a hypergroup automorphism, it follows readily that (2.6) for $\lambda r$ and $f_{\lambda}$ is equivalent to (2.6) for $r$ and $f$. This completes the proof.

2.9 Remark. Eq. (2.3) implies that for $p>2 q-1$ and $(r, a),(s, b) \in \Pi_{q} \times \underline{\mathrm{R}}$ with positive definite matrices $r, s$, the convolution product $\delta_{(r, a)} *_{p, q} \delta_{(s, b)}$ admits a density w.r.t. the Lebesgue measure and hence by the preceding proposition w.r.t. the Haar measure of the hypergroup $\left(\Pi_{q} \times \underline{\mathrm{R}}, *_{p, q}\right)$.

In fact, in the case $p>2 q-1$ consider the linear map

$$
w \mapsto\left(r^{2}+s^{2}+r w s+(r w s)^{*}, \operatorname{Im} \operatorname{tr}(r w s)\right)
$$

from $B_{q} \subset \underline{\mathrm{R}}^{2 q^{2}}$ to $\Pi_{q}^{\circ} \times \underline{\mathrm{R}} \subset R^{q^{2}-1}$ which has a Jacobi matrix with maximal rank $q^{2}-1$. As the square root mapping on the interior $\Pi_{q}^{\circ}$ of $\Pi_{q}$ is a diffeomorphism, the claim follows immediately from the convolution (2.3).

We next turn to the subhypergroups of $\left(\Pi_{q} \times \underline{\mathrm{R}}, *_{p, q}\right)$. Recapitulate for this that a closed set $X \subset \Pi_{q} \times \underline{\mathrm{R}}$ is called a subhypergroup, if for all $x, y \in X$, we have $\bar{x} \in X$ and $\{x\} *\{y\}:=\operatorname{supp}\left(\delta_{x} * \delta_{y}\right) \subset X$. We next determine all subhypergroups of $\left(\Pi_{q} \times \underline{\mathrm{R}}, *_{p, q}\right)$. We begin with examples of subhypergroups.

2.10 Proposition. Let $p>2 q-1, k \in\{1, \ldots, q\}$, and $u \in U_{q}$. Then

$$
X_{k, u}:=\left\{\left(u\left(\begin{array}{cc}
\tilde{r} & 0 \\
0 & 0
\end{array}\right) u^{*}, a\right): \tilde{r} \in \Pi_{k}, a \in \underline{R}\right\}
$$

is a subhypergroup of $\left(\Pi_{q} \times \underline{R}, *_{p, q}\right)$, and the mapping

$$
(\tilde{r}, a) \mapsto\left(u\left(\begin{array}{cc}
\tilde{r} & 0 \\
0 & 0
\end{array}\right) u^{*}, a\right)
$$

is a hypergroup isomorphism between the Heisenberg hpergroup $\left(\Pi_{k} \times \underline{R}, *_{p, k}\right)$ and the subhypergroup $\left(X_{k, u}, *_{p, q}\right)$.

Proof. The $X_{k, I_{q}}$ are obviously subhypergroups by Eq. (2.3). Moreover, using the automorphism $T_{u, 1}$ of Lemma 2.6, we see that the $X_{k, u}$ are subhypergroups for arbitrary $u \in U_{q}$.

In order to check that the subhypergroup $X_{k, u}$ is isomorphic with the hypergroup $\left(\Pi_{k} \times \underline{\mathrm{R}}, *_{p, k}\right)$, we may assume $u=I_{q}$ without loss of generality. Here we first consider the group cases with integer $p \geq 2 q$. Here, the 
inverse image of $X_{k, u}$ under the canonical projection $\varphi: H_{p, q} \rightarrow \Pi_{q} \times \underline{\mathrm{R}}$ is given by the subgroup $\left\{\left(\left(\begin{array}{l}x \\ 0\end{array}\right), a\right), x \in M_{p, k}, a \in \underline{\mathrm{R}}\right\}$ of $H_{p, q}$ which is isomorphic with $H_{p, k}$ and preserved by the action of $U_{p}$. Thus, the preceding construction of the orbit hypergroup structures implies that $\left(X_{k, u}, *_{p, q}\right)$ is isomorphic with $\left(\Pi_{k} \times \underline{\mathrm{R}}, *_{p, k}\right)$ as claimed in this case. Therefore, for integers $p \geq 2 q$ and all $f \in C_{b}\left(\Pi_{q} \times \underline{\mathrm{R}}\right)$ and $(r, a),(s, b) \in \Pi_{k} \times \underline{\mathrm{R}}$,

$$
\delta\left(\left(\begin{array}{cc}
r & 0 \\
0 & 0
\end{array}\right), a\right)^{*_{p, q} \delta}\left(\left(\begin{array}{cc}
s & 0 \\
0 & 0
\end{array}\right), b\right)(f)=\left(\delta_{(r, a)} *_{p, k} \delta_{(s, b)}\right)\left(f_{k}\right)
$$

with $f_{k}(r, a):=f\left(\left(\begin{array}{cc}r & 0 \\ 0 & 0\end{array}\right), a\right)$. If we use the definitions of these convolutions in Theorem 2.4 for arbitrary $p$, analytic continuation via Carlson's theorem yields in the same way as in the proof of Theorem 2.4 that this equation holds for all $p>2 q-1$. This completes the proof.

2.11 Remark. It follows immediately from Eq. (2.3), that $X_{0}:=\{0\} \times \underline{\mathrm{R}}$ is a normal subgroup of $\left(\Pi_{q} \times \underline{\mathrm{R}}, *_{p, q}\right)$ isomorphic with $(\underline{\mathrm{R}},+)$. We now may consider the associated quotient hypergroup $\left(\Pi_{q} \times \underline{\mathrm{R}}, *_{p, q}\right) / X_{0}$ which can be identified with $\Pi_{q}$ obviously in a topological way. Using the definition of the quotient convolution (see e.g. [V2]) as well as Eq. (2.3), this quotient convolution on $\Pi_{q}$ is given by

$$
\left(\delta_{r} * \delta_{s}\right)(f)=\kappa_{p, q} \int_{B_{q}} f\left(\sqrt{r^{2}+s^{2}+r w s+(r w s)^{*}}\right) \cdot \Delta\left(I_{q}-w^{*} w\right)^{p-2 q} d w .
$$

In other words, $\left(\Pi_{q} \times \underline{\mathrm{R}}, *_{p, q}\right) / X_{0}$ is isomorphic with the Bessel hypergroup structure on the cone $\Pi_{q}$ of [R2] with index $p$.

2.12 Lemma. Let $p>2 q-1$. Let $X$ be a subhypergroup of $\left(\Pi_{q} \times \underline{R}, *_{p, q}\right)$ which is not contained in the subgroup $X_{0}$. Then $X_{0} \subset X$.

Proof. Consider a subhypergroup $X \not \subset X_{0}$. Thus there exist $r \in \Pi_{q} \backslash\{0\}$ and $a \in \underline{\mathrm{R}}$ with $(r, a) \in X$. If we restrict the integration in Eq. (2.3) to matrices $w_{c}=(-1 / 2+c i) \cdot I_{q} \in B_{q}$ with $c \in[-\sqrt{3} / 2, \sqrt{3} / 2]$, we conclude from (2.3) and $\sqrt{2 r^{2}+r w_{c} r+r w_{c}^{*} r}=r$ that

$\{r\} \times\left[-\sqrt{3} \cdot \operatorname{tr}\left(r^{2}\right) / 2, \sqrt{3} \cdot \operatorname{tr}\left(r^{2}\right) / 2\right] \subset\{(r, a)\} *_{p, q}\{(r,-a)\} \quad \subset \quad X$.

Therefore, by (2.3), there exists $\varepsilon>0$ such that for all $x \in[-\varepsilon,+\varepsilon]$ we have $(0, x) \in\{(r, x)\} *_{p, q}\{(r,-x)\} \subset X$. As $X_{0}$ is a subgroup isomorphic with $(\underline{\mathrm{R}},+)$, it follows that $X_{0} \subset X$.

2.13 Proposition. Let $X$ be a subhypergroup of $\left(\Pi_{q} \times \underline{R}, *_{p, q}\right)$. Then $X$ is a subgroup of $X_{0}$ or $X$ is equal to one of the subhypergroups $X_{k, u}$ of Proposition 2.10. 
Proof. Let $X$ be a subhypergroup which is not contained in $X_{0}$. Then $X_{0} \subset$ $X$ by Lemma 2.12, and we may consider the quotient subhypergroup $X / X_{0}$ in the quotient hypergroup $\left(\Pi_{q} \times \underline{\mathrm{R}}\right) / X_{0}$ which is isomorphic with the Bessel hypergroup of [R2] on the cone $\Pi_{q}$ with parameter $p$. On the other hand, all subhypergroups of the Bessel hypergroup structures on the $\Pi_{q}$ were classified in Proposition 4.6 of [V3]. As $X_{0} \subset X$, this classification leads immediately to the classification above.

\subsection{Remark. Let}

$$
B:=\left\{y \in \underline{\mathrm{C}}^{q}:\|y\|_{2}<1\right\} \quad \text { and } \quad S:=\left\{y \in \underline{\mathrm{C}}^{q}:\|y\|_{2}=1\right\} .
$$

By Lemma 3.6 and Corollary 3.7 of [R2], the mapping $P: B^{q} \rightarrow B_{q}$ from the direct product $B^{q}$ to the ball $B_{q}$ with

$$
P\left(y_{1}, \ldots, y_{q}\right):=\left(\begin{array}{c}
y_{1} \\
y_{2}\left(I_{q}-y_{1}^{*} y_{1}\right)^{1 / 2} \\
\vdots \\
y_{q}\left(I_{q}-y_{q-1}^{*} y_{q-1}\right)^{1 / 2} \cdots\left(I_{q}-y_{1}^{*} y_{1}\right)^{1 / 2}
\end{array}\right)
$$

establishes a diffeomorphism such that the image of the measure

$$
\Delta\left(I_{q}-w^{*} w\right)^{p-2 q} d w
$$

under $P^{-1}$ is given by $\prod_{j=1}^{q}\left(1-\left\|y_{j}\right\|_{2}^{2}\right)^{p-q-j} d y_{1} \ldots d y_{q}$. Therefore, Eq. (2.3) may be written as

$$
\begin{aligned}
\left(\delta_{(r, a)} *_{p, q} \delta_{(s, b)}\right) & (f) \\
=\kappa_{p, q} & \int_{B^{q}}^{q} f\left(\sqrt{r^{2}+s^{2}+r P(y) s+s P(y)^{*} r}, a+b-\operatorname{Im} \operatorname{tr}(r P(y) s)\right) \\
& \cdot \prod_{j=1}^{q}\left(1-\left\|y_{j}\right\|_{2}^{2}\right)^{p-q-j} d y_{1} \ldots d y_{q}
\end{aligned}
$$

for $p>2 q-1$. Moreover, for $p \rightarrow 2 q-1$, this convolution product converges weakly to the probability measure $\delta_{(r, a)} *_{2 q-1, q} \delta_{(s, b)} \in M^{1}\left(\Pi_{q} \times \underline{\mathrm{R}}\right)$ with

$$
\begin{aligned}
\left(\delta_{(r, a)} *_{2 q-1, q} \delta_{(s, b)}\right)(f) & \\
=\kappa_{2 q-1, q} & \int_{B^{q-1}} \int_{S} f\left(\sqrt{r^{2}+s^{2}+r P(y) s+s P(y)^{*} r}, a+b-\operatorname{Im} \operatorname{tr}(r P(y) s)\right) \\
& \cdot \prod_{j=1}^{q-1}\left(1-\left\|y_{j}\right\|_{2}^{2}\right)^{p-q-j} d y_{1} \ldots d y_{q-1} d \sigma\left(y_{q}\right)
\end{aligned}
$$

where $\sigma \in M^{1}(S)$ is the uniform distribution on $S$ and $\kappa_{2 q-1, q}>0$ a suitable normalization constant.

This convolution is obviously weakly continuous and can be extended to an associative, weakly continuous, and probability preserving convolution 
on $M_{b}\left(\Pi_{q} \times \underline{\mathrm{R}}\right)$ by Theorem 2.4 and taking the limit above. Moreover, all further hypergroup axioms may be also checked readily for (2.9). Finally, the measure $\omega_{2 q-1, q}$ defined as in Proposition 2.8 is a Haar measure of this hypergroup $\left(\Pi_{q} \times \underline{\mathrm{R}}, *_{2 q-1, q}\right)$, the mappings $T_{u, \lambda}(r, a):=\left(\lambda u r u^{*}, \lambda^{2} a\right)$ are also automorphisms here as in Lemma 2.6, and the subsets $X_{k, u} \subset \Pi_{q} \times \underline{\mathrm{R}}$ defined as in Proposition 2.10 are again subhypergroups.

\section{$3 \quad$ Heisenberg-type convolutions associated with Weyl chambers of type B}

In this section we consider the group $U_{q}$ which acts by Lemma 2.6 as a compact group $\left\{T_{u, 1}: u \in U_{q}\right\}$ of automorphisms on the Heisenberg hypergroups $\left(\Pi_{q} \times \underline{\mathrm{R}}, *_{p, q}\right)$. As the orbits of the action of $U_{q}$ on $\Pi_{q}$ by conjugation are described by the ordered eigenvalues $\xi_{1} \geq \xi_{2} \geq \cdots \geq \xi_{q} \geq 0$ of a matrix in $\Pi_{q}$, we may identify the space of all $U_{q}$-orbits of $\left(\Pi_{q} \times \underline{\mathrm{R}}, *_{p, q}\right)$ with the set $\Xi_{q} \times \underline{\mathrm{R}}$ where

$$
\Xi_{q}:=\left\{\xi=\left(\xi_{1}, \ldots \xi_{q}\right) \in \underline{\mathrm{R}}^{q}: \xi_{1} \geq \ldots \geq \xi_{q} \geq 0\right\} .
$$

The set $\Xi_{q}$ is a closed Weyl chamber of the hyperoctahedral group $B_{q}=$ $S_{q} \ltimes \underline{\mathrm{Z}}_{2}^{q}$ which acts on $\underline{\mathrm{R}}^{q}$ by permutations of the basis vectors and sign changes. In this section we show how the convolutions $*_{p, q}$ on $\Pi_{q} \times \underline{\mathrm{R}}$ for $p \geq 2 q-1$ lead to orbit hypergroup convolutions $\circ_{p, q}$ on $\Xi_{q} \times \underline{\mathrm{R}}$ by using methods of [J] or [R3]. In contrast to the hypergroups $\left(\Pi_{q} \times \underline{\mathrm{R}}, *_{p, q}\right)$, the hypergroups $\left(\Xi_{q} \times \underline{\mathrm{R}}, \circ_{p, q}\right)$ are always commutative. We shall identify the characters of these hypergroups in terms of with multivariate Bessel and Laguerre functions associated with the root system $B_{q}$.

Let us go into the details. Let $q \geq 1$ be an integer and $p \in[2 q-1, \infty[$. In the situation described above, the mapping $\Pi_{q} \rightarrow \Xi_{q}, \quad r \mapsto \sigma(r)$, which assigns to each matrix $r$ its ordered spectrum $\sigma(r)$, is continuous, surjective and open w.r.t. the standard topologies on both sets. Therefore the orbit space $\left(\Pi_{q} \times \underline{\mathrm{R}}\right)^{U_{q}}$ (equipped with the quotient topology) may be identified with $\Xi_{q} \times \underline{\mathrm{R}}$ also in a topological way. We now identify both spaces in the obvious way and consider the continuous, surjective and open mapping

$$
\Phi: \Pi_{q} \times \underline{\mathrm{R}} \rightarrow \Xi_{q} \times \underline{\mathrm{R}}, \quad(r, a) \mapsto(\sigma(r), a)
$$

which corresponds to the orbit map above. This mapping is a orbital mapping from the hypergroup $\left(: \Pi_{q} \times \underline{\mathrm{R}}, *_{p, q}\right)$ onto $\Xi_{q} \times \underline{\mathrm{R}}$ in the sense of Section 13 of [J], and it follows readily from Section 13 of $[\mathrm{J}]$ that $\Xi_{q} \times \underline{\mathrm{R}}$ carries a corresponding orbit hypergroup convolution $\circ_{p, q}$ as follows: For $a, b \in \underline{\mathrm{R}}$ and $\xi, \eta \in \Xi_{q}$ we choose representatives $x, y \in \Pi_{q}$ with $\sigma(x)=\xi$ and $\sigma(y)=\eta$ and put

$$
\delta_{(\xi, a)}{ }_{p, q} \delta_{(\eta, b)}:=\Phi\left(\delta_{(x, a)} *_{p, q} \delta_{(y, b)}\right)
$$


The properties of this hypergroup convolution can now be derived similar to Section 4 of [R2]. In particular, we can write down the convolution (3.1) explicitly. For this, we denote the normalized Haar measure on $U_{q}$ by $d u$, and $\xi \in \Xi_{q}$ will always be identified with the diagonal matrix $\operatorname{diag}\left(\xi_{1}, \ldots, \xi_{q}\right) \in$ $\Pi_{q}$ without mentioning.

3.1 Theorem. Let $q \geq 1$ be an integer and $p \in] 2 q-1, \infty\left[\right.$. Then $\Xi_{q} \times \underline{R}$ carries a commutative hypergroup structure with the convolution

$$
\begin{aligned}
& \left(\delta_{(\xi, a)} \circ_{p, q} \delta_{(\eta, b)}\right)(f) \\
& =\kappa_{p, q} \int_{B_{q}} \int_{U_{q}} f\left(\sigma\left(\sqrt{\xi^{2}+u \eta^{2} u^{*}+\xi w u \eta u^{*}+u \eta u^{*} w^{*} \xi}\right),\right. \\
& \left.a+b-\operatorname{Im} \operatorname{tr}\left(\xi w u \eta u^{*}\right)\right) \cdot \Delta\left(I_{q}-w^{*} w\right)^{p-2 q} d u d w
\end{aligned}
$$

for $f \in C_{b}\left(\Xi_{q} \times \underline{R}\right),(\xi, a),(\eta, b) \in \Xi_{q} \times \underline{R}$. The neutral element is given by $(0,0) \in \Xi_{q} \times \underline{R}$, and the involution by $\overline{(\xi, a)}:=(\xi,-a)$.

Moreover, a Haar measure on $\left(\Xi_{q} \times \underline{R}, \circ_{p, q}\right)$ is given by

$$
d \tilde{\omega}_{p, q}(\xi, a):=h_{p, q}(\xi) d \xi d a
$$

with the Lebesgue density

$$
h_{p, q}(\xi):=\prod_{i=1}^{q} \xi_{i}^{2 p-2 q+1} \prod_{i<j}\left(\xi_{i}^{2}-\xi_{j}^{2}\right)^{2} .
$$

Proof. In view of Section 13 of [J] and Section 4 of [R2] on orbit hypergroups, we only have to check the commutativity of $o_{p, q}$ as well as the statement about the Haar measure.

We first turn to the commutativity. We first observe that for integers $p>2 q-1$ by its construction, the hypergroup $\left(\Xi_{q} \times \underline{\mathrm{R}}, \circ_{p, q}\right)$ is isomorphic with the orbit hypergroup which appears when the group $U_{p} \times U_{q}$ acts on the Heisenberg group $H_{p, q}$ by $(u, v)(x, a):=\left(u x v^{*}, a\right)$ for $u \in U_{p}, v \in U_{q}$, $x \in M_{p, q}$ and $a \in \underline{\mathrm{R}}$. Moreover, it is well known that

$$
\left(\left(U_{p} \times U_{q}\right) \ltimes H_{p, q}, U_{p} \times U_{q}\right)
$$

is a Gelfand pair; see [BJR1], $\mathrm{C}$, [F], [Kac]. Therefore, $o_{p, q}$ is commutative for integers $p \geq 2 q$. The general case can now be proved by analytic continuation using Carlson's theorem 2.3 in the same way as in the proof of Theorem 2.4. We omit the details.

On the other hand, we may check commutativity also directly. In fact, let $a, b \in \underline{\mathrm{R}}$ and $\xi, \eta \in \Xi_{q}$. We also regard $\xi, \eta$ as real diagonal matrices as described above. We obtain from invariance of spectrum and trace by 
conjugations that

$$
\begin{aligned}
& \left(\delta_{(\eta, b)}{ }^{\circ}, q, \delta_{(\xi, a)}\right)(f) \\
& =\kappa_{p, q} \int_{B_{q}} \int_{U_{q}} f\left(\sigma\left(\sqrt{u^{*} \eta^{2} u+\xi^{2}+u^{*} \eta w u \xi+\left(u^{*} \eta w u \xi\right)^{*}}\right),\right. \\
& \left.\quad a+b-\operatorname{Im} \operatorname{tr}\left(u^{*} \eta w u \xi\right)\right) \cdot \Delta\left(I_{q}-w^{*} w\right)^{p-2 q} d u d w .
\end{aligned}
$$

Substitution $w \mapsto \bar{w}$ as well as $d w=d \bar{w}, \Delta\left(I_{q}-\bar{w}^{*} \bar{w}\right)=\Delta\left(I_{q}-w^{*} w\right)>0$, $\sigma\left(x^{T}\right)=\sigma(x), \operatorname{tr}\left(x^{T}\right)=\operatorname{tr}(x), \bar{\xi}=\xi$, and $\bar{\eta}=\eta$ imply that this expression is equal to

$$
\begin{gathered}
\kappa_{p, q} \int_{B_{q}} \int_{U_{q}} f\left(\sigma\left(\sqrt{u^{T} \eta^{2} \bar{u}+\xi^{2}+u^{T} \eta w \bar{u} \xi+\xi u^{T} w^{*} \eta \bar{u}}\right)\right. \\
\left.a+b-\operatorname{Im} \operatorname{tr}\left(\xi u^{T} w^{*} \eta \bar{u}\right)\right) \cdot \Delta\left(I_{q}-w^{*} w\right)^{p-2 q} d u d w .
\end{gathered}
$$

Using the substitution $u \mapsto u^{T}$, which preserves the Haar measure on $U_{q}$, as well as the substitution $w \mapsto u^{*} w^{*} u$, which preserves the Lebesgue measure on $B_{q}$, we obtain that the expression above is equal to the right hand side of (3.2). This completes the direct proof of commutativity.

We finally turn to the Haar measure. By Section 13 of [J], the Haar measure $\tilde{\omega}_{p, q} \in M^{+}\left(\Xi_{q} \times \underline{\mathrm{R}}\right)$ is just given as the image of the Haar measure $\omega_{p, q} \in M^{+}\left(\Pi_{q} \times \underline{\mathrm{R}}\right)$ under the projection $\Phi$. As here the second component $\underline{\mathrm{R}}$ is not involved, the computation of this image measure can be carried out in the same way as in the corresponding proof for the matrix Bessel hypergroups in Theorem 4.1 of [R2]. We therefore omit the details.

3.2 Remarks. (1) For $p=2 q-1$ the convolution $*_{2 q-1, q}$ on $\Pi_{q} \times \underline{\mathrm{R}}$ introduced in Section 2.14 can be also transfered to a commutative hypergroup convolution $\circ_{2 q-1, q}$ on $\Xi_{q} \times \underline{\mathrm{R}}$ in the same way as above. We here omit details.

(2) As already mentioned in the preceding theorem, the hypergroups $\left(\Xi_{q} \times\right.$ $\left.\underline{\mathrm{R}}, \circ_{p, q}\right)$ are orbit hypergroups associated with the action of $U_{p} \times U_{q}$ on the Heisenberg group $H_{p, q}$ for integers $p \geq 2 q$. Clearly, one may also form the associated orbit hypergroup structures $\left(\Xi_{q} \times \underline{\mathrm{R}}, \circ_{p, q}\right)$ for all integers $p \geq q$, where then the corresponding convolution for $p=q, q+1, \ldots, 2 q-1$ is degenerated and no longer given by (3.2).

(3) It is clear by the convolution (3.2) that $G:=\{0\} \times \underline{\mathrm{R}}$ is a subgroup of $\left(\Xi_{q} \times \underline{\mathrm{R}}, \circ_{p, q}\right)$ isomorphic with $(\underline{\mathrm{R}},+)$. We thus may form the quotient hypergroup

$$
\left(\Xi_{q} \times \underline{\mathrm{R}}\right) / G:=\left\{G \cdot(\xi, a)=(\xi, \underline{\mathrm{R}}):(\xi, a) \in \Xi_{q} \times \underline{\mathrm{R}}\right\} \simeq \Xi_{q} .
$$

Using this natural identification as well as the canonical projection $\Psi: \Xi_{q} \times \underline{\mathrm{R}} \rightarrow \Xi_{q}$, the quotient convolution is defined by

$$
\delta_{\xi} \bullet_{p, q} \delta_{\eta}:=\Psi\left(\delta_{(\xi, 0)} o_{p, q} \delta_{(\eta, 0)}\right),
$$


i.e.,

$$
\begin{gathered}
\left(\delta_{\xi} \circ_{p, q} \delta_{\eta}\right)(f) \\
=\kappa_{p, q} \int_{B_{q}} \int_{U_{q}} f\left(\sigma\left(\sqrt{\xi^{2}+u \eta^{2} u^{*}+\xi w u \eta u^{*}+u \eta u^{*} w^{*} \xi}\right)\right) \\
\cdot \Delta\left(I_{q}-w^{*} w\right)^{p-2 q} d u d w
\end{gathered}
$$

for $f \in C_{b}\left(\Xi_{q}\right), \xi, \eta \in \Xi_{q}$. In other words, the quotient hypergroup $\left(\left(\Xi_{q} \times \underline{\mathrm{R}}\right) / G, \bullet_{p, q}\right)$ is precisely the Bessel-hypergroup on the Weylchamber $\Xi_{q}$ as studied in Section 4 of [R2] for the field $\underline{\mathrm{C}}$, i.e., the parameter $d=2$ there.

We next turn to the characters of the commutative hypergroups $\left(\Xi_{q} \times\right.$ $\left.\underline{\mathrm{R}}, \circ_{p, q}\right)$. For this we first recapitulate some basic notions and facts about commutative hypergroups mainly from [J] and [BH].

3.3 Some facts and notions on commutative hypergroups. Let $(X, *)$ be a commutative hypergroup. Then there is a Haar measure $\omega \in M^{+}(X)$ which is unique up to a multiplicative constant. We introduce the dual space

$$
\hat{X}:=\left\{\alpha \in C_{b}(X): \quad \delta_{x} * \delta_{\bar{y}}(\alpha)=\alpha(x) \overline{\alpha(y)} \quad \text { for all } x, y \in X\right\}
$$

and the space of all multiplicative functions

$$
\chi_{b}(X):=\left\{\alpha \in C_{b}(X): \quad \delta_{x} * \delta_{y}(\alpha)=\alpha(x) \alpha(y) \quad \text { for all } x, y \in X\right\},
$$

and equip both with the topology of locally uniform convergence. Both spaces are locally compact, and for a Gelfand pair $(G, K)$, the space of spherical functions corresponds to the space $\chi_{b}(X)$ for the double coset hypergroup $(G / / K, *)$. The elements of $\hat{X}$ are called characters.

We define the Fourier transform $.^{\wedge}: L^{1}(X, \omega) \rightarrow C_{0}(\hat{X})$ with

$$
\hat{f}(\alpha):=\int_{X} \overline{\alpha(x)} \cdot f(x) d \omega(x) .
$$

Then there exists a unique Plancherel measure $\pi \in M^{+}(\hat{X})$ such that the Fourier transform becomes an $L^{2}$-isometry, i.e., for all $f \in L^{1}(X, \omega) \cap$ $L^{2}(X, \omega)$ we have $\int_{X}|f|^{2} d \omega=\int_{\hat{X}}|\hat{f}|^{2} d \pi$, and the Fourier transform can be extended to an isometric isomorphism between $L^{2}(X, \omega)$ and $L^{2}(\hat{X}, \pi)$.

Different to the case of abelian groups, it may occur that $\operatorname{supp} \pi \neq$ $\hat{X} \neq \chi_{b}(X)$. This is the case for instance for Gelfand pairs associated with noncompact semisimple Lie groups. On the other hand, there is a growth criterion in hypergroup theory which ensures supp $\pi=\hat{X}=\chi_{b}(X)$. To explain this, take a compact set $A \subset X$ and define recursively the sets $A^{(n)}$ by $A^{(1)}=A$ and $A^{(n+1)}=A^{(n)} * A^{(1)}=\bigcup_{x \in A^{(n)}, y \in A^{(1)}} \operatorname{supp}\left(\delta_{x} * \delta_{y}\right)$. We say that $(X, *)$ has subexponential growth if for all compact sets $A \subset X$ and all $c>1$ we have $\omega\left(A^{(n)}\right)=o\left(c^{n}\right)$ for $n \rightarrow \infty$. It was proved in Vog and [V1] that for each commutative hypergroup $(X, *)$ with subexponential growth, $\operatorname{supp} \pi=\hat{X}=\chi_{b}(X)$. 
We now return to the hypergroups $\left(\Xi_{q} \times \underline{\mathrm{R}}, o_{p, q}\right)$. As the Heisenberg groups have polynomial growth, the following result is not surprising:

3.4 Lemma. The hypergroups $\left(\Xi_{q} \times \underline{R}, \circ_{p, q}\right)$ have subexponential growth for $p \geq 2 q-1$.

Proof. We see from (3.2) that for all $(\xi, a),(\eta, b) \in \Xi_{q} \times \underline{\mathrm{R}}$ and $(\tau, c) \in$ $\operatorname{supp}\left(\delta_{(\xi, a)} \circ_{p, q} \delta_{(\eta, b)}\right)$ the first (and thus largest) components of the vectors $\tau, \xi, \eta$ satisfy $\tau_{1} \leq \xi_{1}+\eta_{1}$ and $|c| \leq|a|+|b|+\xi_{1} \eta_{1}$. Now let $C \subset \Xi_{q} \times \underline{\mathrm{R}}$ be compact. Choose $d>0$ such that $\xi_{1} \leq d$ and $|a| \leq d$ for all $(\xi, a) \in C$. A simple induction shows that then for all $n \in \mathbb{N}$ and all $(\tau, c) \in C^{(n)}$ we have $0 \leq \tau_{q} \leq \ldots \leq \tau_{1} \leq n d$ and $|c| \leq n d+\frac{n(n-1)}{2} \cdot d^{2}$. As the Haar measure $\tilde{\omega}_{p, q}$ has a polynomially growing Lebesgue density by Theorem 3.1, the assertion is clear.

By the results of Section 3.3 we obtain:

3.5 Corollary. The hypergroups $\left(\Xi_{q} \times \underline{R}, o_{p, q}\right)$ satisfy supp $\pi=\hat{X}=\chi_{b}(X)$.

Consider the canonical projection $\Phi: \Pi_{q} \times \underline{\mathrm{R}} \rightarrow \Xi_{q} \times \underline{\mathrm{R}}$ as in the beginning of this section. This mapping is an orbital morphism in the sense of [J], and we conclude from $[\mathrm{J}]$ :

3.6 Corollary. Let $p \geq 2 q-1$. For each character $\alpha$ of $\left(\Xi_{q} \times \underline{R}, \circ_{p, q}\right)$, the function $\alpha \circ \Phi \in C_{b}\left(\Pi_{q} \times \underline{R}\right)$ is positive definite on the hypergroup $\left(\Pi_{q} \times \underline{R}, *_{p, q}\right)$.

We next introduce a set $\Sigma_{p, q}$ of characters of the hypergroups $\left(\Xi_{q} \times\right.$ $\left.\underline{\mathrm{R}}, \circ_{p, q}\right)$. Later on we shall see that this set in fact consists of all characters. This set $\Sigma_{p, q}$ consists of two disjoint sets $\Sigma_{p, q}^{1}$ and $\Sigma_{p, q}^{2}$ of functions where these functions are described in terms of multivariate Laguerre and Bessel functions respectively as discussed in [FK]. This is not surprising, as this connection is well-known for $q=1$ (see the product formula in [Ko], [T] and references cited there) as well as for the group cases with integers $p, q \geq$ 1; see $[\mathrm{F}]$ and references there. Before going into details, we collect some notions and facts from $[\mathrm{BF},[\mathrm{F}],[\mathrm{FK}]$, and $[\mathrm{Kan}$. We start with some basic notions on multivariate special functions:

3.7 Spherical polynomials. Let $\mathbf{m}=\left(m_{1}, \ldots, m_{q}\right)$ be a partition of length $q$ with integers $m_{1} \geq m_{2} \geq \ldots \geq m_{q} \geq 0$. We define its length $|\mathbf{m}|:=m_{1}+\ldots+m_{q}$, the generalized Pochhammer symbol

$$
(x)_{\mathbf{m}}=\prod_{j=1}^{q}(x-j+1)_{m_{j}}
$$

for $x \in \underline{\mathrm{R}}$ (note that we here always use $d=2$ in the notion of [FK]), as well as the dimension constant

$$
d_{\mathbf{m}}:=\frac{(p)_{\mathbf{m}}(q)_{\mathbf{m}}}{h(\mathbf{m})^{2}}
$$


where $h(\mathbf{m})$ is the product of the hook lengths of $\mathbf{m}$; see pp. 237 of $[\mathbf{F}]$ and p. 66 of [M2]. Moreover, for partitions $\mathbf{m}$ we define the spherical polynomials

$$
\Phi_{\mathbf{m}}(x)=\int_{U_{q}} \Delta_{\mathbf{m}}\left(u x u^{-1}\right) d u \quad \text { for } \quad x \in M_{q, q}
$$

where $d u$ is the normalized Haar measure of $U_{q}, \Delta_{\mathbf{m}}$ is the power function

$$
\Delta_{\mathbf{m}}(x):=\Delta_{1}(x)^{m_{1}-m_{2}} \Delta_{2}(x)^{m_{2}-m_{3}} \cdot \ldots \cdot \Delta_{q}(x)^{m_{q}},
$$

and the $\Delta_{i}(x)$ are the principal minors of the determinant $\Delta(x)$, see Ch. XI of [FK] for details. The $\Phi_{\mathbf{m}}$ are homogeneous of degree $|\mathbf{m}|$ and satisfy $\Phi_{\mathbf{m}}(0)=0$ for $\mathbf{m} \neq \mathbf{0}, \Phi_{0}(0)=1$, and $\Phi_{\mathbf{m}}\left(I_{q}\right)=1$ for the identity matrix $I_{q} \in \underline{\mathrm{C}}^{q, q}$.

We also consider the renormalized, so-called zonal polynomials $Z_{\mathbf{m}}=$ $c_{\mathbf{m}} \Phi_{\mathbf{m}}$ with the constants

$$
c_{\mathbf{m}}:=\frac{(q)_{\mathbf{m}}|\mathbf{m}| !}{h(\mathbf{m})^{2}}>0 .
$$

This normalization is characterized by

$$
(\operatorname{tr} x)^{k}=\sum_{|\mathbf{m}|=k} Z_{\mathbf{m}}(x) \quad \text { for } k \in \underline{N}_{0} .
$$

In fact, the normalization constant $c_{\mathbf{m}}$ can be easily derived from (3.7) and some formulas on pp. 237-239 of [F]; see also Section XI.5 of [FK] or [Kan]. Clearly, we have $Z_{\mathbf{m}}\left(I_{q}\right)=c_{\mathbf{m}}$.

By construction, the $\Phi_{\mathbf{m}}$ and $Z_{\mathbf{m}}$ are invariant under conjugation by $U_{q}$ and thus depend only on the eigenvalues of their argument. More precisely, for Hermitian $x \in M_{q, q}$ with eigenvalues $\xi=\left(\xi_{1}, \ldots, \xi_{q}\right) \in \underline{\mathrm{R}}^{q}$, we have $Z_{\mathbf{m}}(x)=C_{\mathbf{m}}^{1}(\xi)$ for the Jack polynomials $C_{\lambda}^{1}$ (c.f. [FK], [R2]). They are homogeneous of degree $|\mathbf{m}|$ and symmetric in their arguments.

We also introduce the generalized binomial coefficients $\left(\begin{array}{c}\mathbf{m} \\ \mathbf{n}\end{array}\right)$ for partitions $\mathbf{m}, \mathbf{n}$ by the unique expansion

$$
\Phi_{\mathbf{m}}\left(I_{q}+x\right)=\sum_{|\mathbf{n}| \leq|\mathbf{m}|}\left(\begin{array}{c}
\mathbf{m} \\
\mathbf{n}
\end{array}\right) \Phi_{\mathbf{n}}(x)
$$

with the identity matrix $I_{q} \in \underline{\mathrm{C}}^{q, q}$. These Binomial coefficients satisfy $\left(\begin{array}{l}\mathbf{m} \\ \mathbf{n}\end{array}\right) \neq$ 0 only for $\mathbf{n} \subset \mathbf{m}$, i.e. for $n_{i} \leq m_{i}$ for $i=1, \ldots, q$. Moreover, it follows from [L] that $\left(\begin{array}{l}\mathbf{m} \\ \mathbf{n}\end{array}\right) \geq 0$, and that for integers $k$,

$$
\sum_{|\mathbf{n}|=k}\left(\begin{array}{c}
\mathbf{m} \\
\mathbf{n}
\end{array}\right)=\left(\begin{array}{c}
|\mathbf{m}| \\
k
\end{array}\right)
$$


3.8 Multivariate Laguerre polynomials. According to p. 343 of [FK] we define the multivariate Laguerre polynomials

$$
L_{\mathbf{m}}^{p}(x):=\sum_{|\mathbf{n}| \leq|\mathbf{m}|}\left(\begin{array}{c}
\mathbf{m} \\
\mathbf{n}
\end{array}\right) \frac{(p)_{\mathbf{m}}}{(p)_{\mathbf{n}}} \cdot \Phi_{\mathbf{n}}(-x)
$$

and the associated multivariate Laguerre functions

$$
l_{\mathbf{m}}^{p}(x):=e^{-\operatorname{tr}(x)} L_{\mathbf{m}}^{p}(2 x)
$$

for $x \in \underline{\mathrm{C}}^{q, q}$. The functions $L_{\mathbf{m}}^{p}$ and $l_{\mathbf{m}}^{p}$ are also invariant under conjugation by $U_{q}$ and may thus be regarded as functions in their eigenvalues, i.e., as functions on $\Xi_{q}$. We shall do this from now on without separate notation.

With a slight change of notation, these Laguerre polynomials and functions are also considered by Baker and Forrester $[\mathrm{BF}$ in the context of Calogero-Sutherland models and Dunkl operators. In fact, a comparison of the notions in $[\mathrm{FK}]$ and $[\mathrm{BF}]$ shows that our polynomials $L_{\mathbf{m}}^{p}(x)$ defined above agree with the Laguerre polynomials $|\mathbf{m}| ! \cdot L_{\mathbf{m}}^{p-q}(x ; 1)$ in the notion of Proposition 4.3 of $[\mathrm{BF}]$ :

$$
L_{\mathbf{m}}^{p}(x)=|\mathbf{m}| ! \cdot L_{\mathbf{m}}^{p-q}(x ; 1) \quad(\text { in the sense of }[\mathrm{BF}]) .
$$

We next collect some known properties about these Laguerre polynomials:

3.9 Lemma. The polynomials $L_{\mathbf{m}}^{p}(x)$ form an orthogonal basis on the Hilbert space $L^{2}\left(\Xi_{q}, d \mu_{p, q}\right)$ with the measure

$$
d \mu_{p, q}(x):=\prod_{i=1}^{q}\left(e^{-x_{i}} x_{i}^{p-q}\right) \cdot \prod_{i<j}\left(x_{i}-x_{j}\right)^{2} d x .
$$

Moreover, for each partition $\mathbf{m}$,

$$
\int_{\Xi_{q}}\left(L_{\mathbf{m}}^{p}(x)\right)^{2} d \mu_{p, q}(x)=d_{p, q} \cdot \frac{|\mathbf{m}| !(p)_{\mathbf{m}}}{q ! c_{\mathbf{m}}}=d_{p, q} \cdot \frac{(p)_{\mathbf{m}} h(\mathbf{m})^{2}}{q !(q)_{\mathbf{m}}}
$$

with the normalization constant

$$
d_{p, q}:=\mu_{p, q}\left(\Xi_{q}\right)=\int_{\Xi_{q}} 1 d \mu_{p, q}(x)
$$

Finally, $L_{\mathbf{m}}^{p}(0)=(p)_{\mathbf{m}}$.

Proof. For the orthogonality and the normalization we refer to Corollary XV.4.3 of [FK] or Proposition 4.10 of $\left[\mathrm{BF}\right.$. As the $L_{\mathbf{m}}^{p}$ form a basis of all polynomials in $q$ dimensions (use e.g. Proposition 4.3 of $[\mathrm{BF}]$ and the fact that the Jack polynomials form a basis), the completeness of the system $\left(L_{\mathbf{m}}^{p}\right)_{\mathbf{m}}$ can be derived by a classical Fourier argument like in the onedimensional case for Laguerre polynomials. Another possibility here is to use results of $\mathrm{dJ}$. 
We next turn to multivariate Bessel functions of two arguments $\xi, \eta \in \underline{\mathrm{C}}^{q}$ :

3.10 Multivariate Bessel functions. According to Kaneko Kan (see also Section 2.2 of [R2]) we put

$$
J_{p}(\xi, \eta):=\sum_{\mathbf{m}} \frac{(-1)^{|\mathbf{m}|}}{(p)_{\mathbf{m}}|\mathbf{m}| !} \cdot \frac{C_{\mathbf{m}}^{1}(\xi) C_{\mathbf{m}}^{1}(\eta)}{C_{\mathbf{m}}^{1}(1, \ldots, 1)}
$$

For $\eta \in \Xi_{q}$ we now define the functions $\psi_{\eta}^{p} \in C_{b}\left(\Xi_{q} \times \underline{\mathrm{R}}\right)$ by

$$
\psi_{\eta}^{p}(\xi, t):=J_{p}\left(\xi^{2} / 2, \eta^{2} / 2\right)
$$

according to Section 4.2 of [R2]. We denote the set of all $\psi_{\eta}^{p}$ with $\eta \in \Xi_{q}$ by $\Sigma_{p, q}^{2}$.

The multivariate Bessel functions appear as limits of the Laguerre functions above. For the group case with integers $p$, this was observed already by Faraut [F].

3.11 Lemma. Let $p \geq 2 q-1$, and let $\left(\mathbf{m}_{k}\right)_{k}$ be a sequence of partitions and $\left(\lambda_{k}\right)_{k} \subset \mathbb{R} \backslash\{0\}$ a sequence with $\lambda_{k} \rightarrow 0$ and $\lim _{k \rightarrow \infty} \lambda_{k} \cdot \mathbf{m}_{k}=\eta \in \Xi_{q}$. Then, for $p \geq 2 q-1$,

$$
\lim _{k \rightarrow \infty} \frac{L_{\mathbf{m}_{k}}^{p}\left(\left|\lambda_{k}\right| \xi^{2}\right)}{L_{\mathbf{m}_{k}}^{p}(0)}=J_{p}\left(\xi^{2} / 2, \eta^{2} / 2\right)
$$

uniformly on compact subsets for $\xi \in \Xi_{q}$.

Proof. Writing the expansions of the Laguerre polynomials and Bessel functions above in terms of so-called shifted Schur functions precisely as on pp. 240-241 of [F], it can be checked as in Proposition 3.3 of [F] that for all partitions $\mathbf{n}$, the coefficients of the expansion of $\frac{L_{\mathbf{m}_{k}}^{p}\left(\left|\lambda_{k}\right| \xi^{2}\right)}{L_{\mathbf{m}_{k}}^{p}(0)}$ tend to the corresponding coefficients of $J_{p}\left(\xi^{2} / 2, \eta^{2} / 2\right)$. Moreover, as $\Phi_{\mathbf{n}}$ is homogeneous of degree $|\mathbf{n}|$ with $\left|\Phi_{\mathbf{n}}(x)\right| \leq 1$ for $\|x\|_{2}=1$, and as

$$
(p)_{\mathbf{n}} \geq(q)_{n_{1}} \cdots(q)_{n_{q}} \geq\left((q)_{\lfloor|\mathbf{n}| / q\rfloor}\right)^{q} \quad \text { for } \quad p \geq 2 q-1
$$

we obtain with Eq. (3.8) that

$$
\begin{aligned}
\left|\frac{L_{\mathbf{m}_{k}}^{p}\left(\left|\lambda_{k}\right| \xi^{2}\right)}{L_{\mathbf{m}_{k}}^{p}(0)}\right| & \leq \sum_{\mathbf{n}}\left(\begin{array}{c}
\mathbf{m}_{k} \\
\mathbf{n}
\end{array}\right) \frac{1}{(p)_{\mathbf{n}}} \cdot\left|\Phi_{\mathbf{n}}\left(\xi^{2}\right)\right| \cdot\left|\lambda_{k}\right|^{|\mathbf{n}|} \\
& \leq \sum_{j} \sum_{|\mathbf{n}|=j}\left(\begin{array}{c}
\mathbf{m}_{k} \\
\mathbf{n}
\end{array}\right) \frac{1}{\left((q)_{\lfloor j / q\rfloor}\right)^{q}} \cdot\left|\lambda_{k}\right|^{j}\|\xi\|^{2 j} \\
& \leq \sum_{j} \frac{\left(\left|\mathbf{m}_{k}\right|\left|\lambda_{k}\right|\right)^{j}}{j ! \cdot\left((q)_{\lfloor j / q\rfloor}\right)^{q}} \cdot\|\xi\|^{2 j}<\infty
\end{aligned}
$$

locally uniformly for $\xi \in \Xi$. This readily implies the claimed locally uniform convergence. 
Let us return to the characters of the hypergroups $\left(\Xi_{q} \times \underline{\mathrm{R}}, \circ_{p, q}\right)$.

3.12 Definition. For $\lambda \in \underline{\mathrm{R}} \backslash\{0\}$ and a partition $\mathbf{m}$, we define the function $\varphi_{\lambda, \mathbf{m}}^{p} \in C_{b}\left(\Xi_{q} \times \underline{\mathrm{R}}\right)$ by

$$
\varphi_{\lambda, \mathbf{m}}^{p}(\xi, t):=e^{i \lambda t} \cdot \frac{l_{\mathbf{m}}^{p}\left(|\lambda| \xi^{2} / 2\right)}{l_{\mathbf{m}}^{p}(0)}=e^{i \lambda t} \cdot e^{-|\lambda|\left(\xi_{1}^{2}+\ldots \xi_{q}^{2}\right) / 2} \cdot \frac{L_{\mathbf{m}}^{p}\left(|\lambda| \xi^{2}\right)}{L_{\mathbf{m}}^{p}(0)}
$$

with $\xi^{2}:=\left(\xi_{1}^{2}, \ldots, \xi_{q}^{2}\right)$. We denote the set of all $\varphi_{\lambda, \mathbf{m}}^{p}$ with $\lambda \in \underline{\mathrm{R}} \backslash\{0\}$ and partitions $\mathbf{m}$ by $\Sigma_{p, q}^{1}$.

We notice that for integers $p$, the functions in the set $\Sigma_{p, q}^{1}$ agree with the spherical functions $\varphi(\lambda, \mathbf{m}, . .$.$) of pp. 238-241 of Faraut [F] where there the$ Laguerre polynomials are defined with some parameter shift.

Furthermore, we denote the set of all Bessel functions $\psi_{\eta}^{p}$ with $\eta \in \Xi_{q}$ by $\Sigma_{p, q}^{2}$. Again, for integers $p \geq 1$, the set $\Sigma_{p, q}^{2}$ consists of spherical functions by $[\mathrm{F}]$. We note that Faraut (p. 241 of $[\mathrm{F}]$ ) uses a slightly different notion for these Bessel functions; in his notion we have

$$
\psi(\eta, \xi)=J_{p}\left(\xi^{2}, \eta\right)=\psi_{\sqrt{2 \eta}}^{p}(\sqrt{2} \cdot \xi, t) \quad(t \in \underline{\mathrm{R}} \quad \text { arbitrary }) .
$$

3.13 Theorem. Let $p \geq 2 q-1$. Then all functions in $\Sigma_{p, q}:=\Sigma_{p, q}^{1} \cup \Sigma_{p, q}^{2}$ are characters of the hypergroup $\left(\Xi_{q} \times \underline{R}, \circ_{p, q}\right)$.

Proof. By Corollary 3.5, it suffices to show that all functions in $\Sigma_{p, q}$ are multiplicative and bounded. Taking Remark 3.2(3) and the results of Section 4 of [R2] into account, this is clear for all functions in $\Sigma_{p, q}^{2}$.

The proof for the Laguerre functions in $\Sigma_{p, q}^{1}$ is slightly more involved. Here we first consider the group cases with integers $p \geq 2 q-1$ where $\left(\Xi_{q} \times\right.$ $\left.\underline{\mathrm{R}}, \circ_{p, q}\right)$ is isomorphic with the orbit hypergroup which appears when the group $U_{p} \times U_{q}$ acts on the Heisenberg group $H_{p, q}$. In this cases it is well known that the functions in $\Sigma_{p, q}^{1}$ correspond to bounded spherical functions on the associated Gelfand pairs; see $[\mathrm{F}]$ and references cited there. For general parameters $p>2 q-1$ we again employ analytic continuation by Carlson's theorem 2.3. For this we fix a partition $\mathbf{m}, \lambda \in \underline{\mathrm{R}}^{\times}$, and $(\xi, a),(\eta, b) \in \Xi_{q} \times \underline{\mathrm{R}}$, and consider the function

$$
\begin{aligned}
F(p):= & \varphi_{\lambda, \mathbf{m}}^{p}(\xi, a) \cdot \varphi_{\lambda, \mathbf{m}}^{p}(\eta, b) \\
& -\kappa_{p, q} \int_{B_{q}} \int_{U_{q}} \varphi_{\lambda, \mathbf{m}}^{p}\left(\sigma\left(\sqrt{\xi^{2}+u \eta^{2} u^{*}+\xi w u \eta u^{*}+u \eta u^{*} w^{*} \xi}\right)\right. \\
& \left.a+b-\operatorname{Im} \operatorname{tr}\left(\xi w u \eta u^{*}\right)\right) \cdot \Delta\left(I_{q}-w^{*} w\right)^{p-2 q} d u d w
\end{aligned}
$$

which has zeros for integer values $p \geq 2 q$. Moreover, $F$ is analytic on $W:=$ $\{p \in \underline{\mathrm{C}}: \operatorname{Re} p>2 q-1\}$. Furthermore, because of

$$
\left|(p)_{\mathbf{m}}\right|=\left|\prod_{j=1}^{q}(p-j+1)_{m_{j}}\right| \geq 1
$$


for $p \in W$ and the definition of the Laguerre functions $\varphi_{\lambda, \mathbf{m}}^{p}$ above, we see that $\varphi_{\lambda, \mathrm{m}}^{p}$ remains bounded for $p \in W$ locally uniformly for $(\xi, a) \in \Xi_{q} \times \underline{\mathrm{R}}$. Therefore, for a suitable constant $C>0$,

$$
|F(p)| \leq C^{2}+C \cdot\left|\kappa_{p, q}\right| \int_{B_{q}} \int_{U_{q}}\left|\Delta\left(I_{q}-w^{*} w\right)^{p-2 q}\right| d u d w \leq C^{2}+C^{2} \cdot\left|\kappa_{p, q}\right|
$$

with $\left|\kappa_{p, q}\right|=O\left(|p|^{q^{2}}\right)$ by (2.4) for $p \in W$. Therefore, by Theorem 2.3, $F(p)=0$ for all $p>2 q-1$ which proves that all elements of $\Sigma_{p, q}^{1}$ are multiplicative for all $p \geq 2 q-1$. We finally note that the Laguerre functions in $\Sigma_{p, q}^{1}$ are obviously bounded by their definition.

As bounded multiplicative functions $\alpha$ on a commutative hypergroup satisfy $\|\alpha\|_{\infty}=1$, we obtain:

3.14 Corollary. For all $p \geq 2 q-1, \lambda \in \underline{R} \backslash\{0\}$ and all partitions $\mathbf{m}$, $\left\|\varphi_{\lambda, \mathbf{m}}^{p}\right\|_{\infty}=1$

We next turn to the Plancherel measures $\pi_{p, q}$ of the hypergroups $\left(\Xi_{q} \times\right.$ $\left.\underline{\mathrm{R}}, \circ_{p, q}\right)$. These measures are well-known for the group cases with integers $p$ by [BJRW], BJR2], [F], as well as for $q=1$ in the general case; see e.g. Section 8.1 of [T].

3.15 Theorem. Let $p \geq 2 q-1$. If the hypergroup $\left(\Xi_{q} \times \underline{R}, \circ_{p, q}\right)$ is equipped with the Haar measure $\tilde{\omega}_{p, q}$ according to Theorem 3.1, then the associated Plancherel measure $\pi_{p, q} \in M^{+}\left(\Sigma_{p, q}\right)$ according to Section 3.3 is given by

$$
\pi_{p, q}(g)=\frac{2^{q} \cdot q !}{d_{p, q} \cdot 2 \pi} \cdot \int_{\underline{R} \backslash\{0\}} \sum_{\mathbf{m}} \frac{(p)_{\mathbf{m}}(q)_{\mathbf{m}}}{h(\mathbf{m})^{2}} \cdot g(\mathbf{m}, \lambda)|\lambda|^{p q} d \lambda
$$

for $g \in L^{1}\left(\Sigma_{p, q}, \pi_{p, q}\right)$ where clearly the set $\Sigma_{p, q}$ of functions is identified with its describing parameter set in the obvious way.

Proof. Let $f \in C_{c}\left(\Xi_{q} \times \underline{\mathrm{R}}\right)$. The classical Plancherel formula for $(\underline{\mathrm{R}},+)$ says that the classical Fourier transform

$$
F(x, \lambda):=\int_{\underline{\mathbf{R}}} f(x, t) e^{-i \lambda t} d t
$$

w.r.t. the variable $t$ satisfies

$$
\|f\|_{2, \tilde{\omega}_{p, q}}^{2}=\frac{1}{2 \pi} \cdot \int_{\Xi_{q}}\left(\int_{\underline{\mathbb{R}}}|F(x, \lambda)|^{2} d \lambda\right) h_{p, q}(x) d x .
$$

For fixed $\lambda \in \underline{R} \backslash\{0\}$, we now consider the renormalized Laguerre functions

$$
\tilde{\varphi}_{|\lambda|, \mathbf{m}}^{p}(z):=l_{\mathbf{m}}^{p}\left(|\lambda| z^{2} / 2\right) / l_{\mathbf{m}}^{p}(0)
$$


which satisfy $\varphi_{\lambda, \mathbf{m}}^{p}(z, t)=e^{i \lambda t} \tilde{\varphi}_{|\lambda|, \mathbf{m}}^{p}(z)$. We then obtain with the Haar density (3.3), the transformation formula, and $h_{p, q}(c x)=c^{2 p q-q} \cdot h_{p, q}(x)$ for $c>0$ that

$$
c_{\lambda, \mathbf{m}}:=\int_{\Xi_{q}}\left|\tilde{\varphi}_{|\lambda|, \mathbf{m}}^{p}(x)\right|^{2} h_{p, q}(x) d x=\frac{1}{|\lambda|^{p q}} \int_{\Xi_{q}} \frac{l_{\mathbf{m}}^{p}\left(z^{2} / 2\right)^{2}}{l_{\mathbf{m}}^{p}(0)^{2}} h_{p, q}(z) d z .
$$

Moreover, according to Lemma [3.9, the functions $\tilde{\varphi}_{1, \mathbf{m}}^{p} / \sqrt{c_{1, \mathbf{m}}}$ form an orthonormal basis of $L^{2}\left(\Xi_{q}, h_{p, q}(x) d x\right)$. Therefore, by Parseval's identity,

$$
\|f\|_{2, \tilde{\omega}_{p, q}}^{2}=\frac{1}{2 \pi} \sum_{\mathbf{m} \geq \mathbf{0}} \int_{\underline{\mathrm{R}}}\left|\int_{\Xi_{q}} F(x, \lambda) \overline{\tilde{\varphi}_{|\lambda|, \mathbf{m}}^{p}(x)} \cdot h_{p, q}(x) d x\right|^{2} \cdot \frac{1}{c_{\lambda, \mathbf{m}}} d \lambda
$$

As by Section 3.3 and Theorem 3.1 the hypergroup Fourier transform $\hat{f}$ is given by

$$
\begin{aligned}
\hat{f}(\lambda, \mathbf{m}) & =\int_{\Xi_{q}} \int_{\underline{\mathrm{R}}} \overline{\varphi_{|\lambda|, \mathbf{m}}^{p}(x)} \cdot f(x, t) d t h_{p, q}(x) d x \\
& =\int_{\Xi_{q}} F(x, \lambda) \tilde{\varphi}_{|\lambda|, \mathbf{m}}^{p}(x) \cdot h_{p, q}(x) d x,
\end{aligned}
$$

we obtain from Lemma 3.9 that

$$
\begin{aligned}
\|f\|_{2, \tilde{\omega}_{p, q}}^{2} & =\frac{1}{2 \pi} \sum_{\mathbf{m} \geq \mathbf{0}} \int_{\underline{\mathrm{R}}}|\hat{f}(\lambda, \mathbf{m})|^{2} \cdot \frac{1}{c_{\lambda, \mathbf{m}}} d \lambda \\
& =\frac{2^{q} \cdot q !}{d_{p, q} \cdot 2 \pi} \cdot \sum_{\mathbf{m} \geq \mathbf{0}} \int_{\underline{\mathrm{R}}}|\hat{f}(\lambda, \mathbf{m})|^{2} \cdot|\lambda|^{p q} d \lambda \cdot \frac{(p)_{\mathbf{m}}(q)_{\mathbf{m}}}{h(\mathbf{m})^{2}} \\
& =\|\hat{f}\|_{2, \pi_{p, q}}^{2}
\end{aligned}
$$

with the measure $\pi_{p, q}$ introduced in the theorem. As $C_{c}\left(\Xi_{q} \times \underline{\mathrm{R}}\right)$ is dense in $L^{2}\left(\Xi_{q} \times \underline{\mathrm{R}}, h_{p, q}(x) d x d t\right)$, the equation holds also for all $f \in L^{2}\left(\Xi_{q} \times\right.$ $\left.\underline{\mathrm{R}}, h_{p, q}(x) d x d t\right)$, which caracterizes the Plancherel measure as claimed.

We next prove that the characters in $\Sigma_{p, q}$ form the complete dual space $\hat{X}$ of $\left(\Xi_{q} \times \underline{\mathrm{R}}, \circ_{p, q}\right)$. The following representation of the topology on $\hat{X}$ as a Heisenberg fan is due to J. Faraut who considered the group case with integer $p$ :

3.16 Theorem. Let $p \geq 2 q-1$. Then the set $\Sigma_{p, q}$ is equal to the complete dual space $\hat{X}$ of the hypergroup $\left(\Xi_{q} \times \underline{R}, o_{p, q}\right)$. More precisely, if the closed subset

$$
D:=\left(\{0\} \times \Xi_{q}\right) \cup\{(\lambda, \lambda \cdot \mathbf{m}): \lambda \in \underline{R} \backslash\{0\}, \mathbf{m} \text { a partition }\}
$$

of $\underline{R}^{q+1}$ carries the usual topology as a subset of $\underline{R}^{q+1}$, and if $\hat{X}=\Sigma_{p, q}$ carries the topology of locally uniform convergence, then the mapping

$$
E: \Sigma_{p, q} \rightarrow D
$$


with

$$
\varphi_{\lambda, \mathbf{m}}^{p} \in \Sigma_{p, q}^{1} \mapsto\left(\lambda, \lambda m_{1}, \ldots, \lambda m_{q}\right)
$$

and

$$
\psi_{\rho}^{p} \in \Sigma_{p, q}^{2} \mapsto\left(0, \rho_{1}, \ldots, \rho_{q}\right)
$$

establishes an homeomorphism.

Proof. We first notice that it follows easily from the definition of the functions $\varphi_{\lambda, \mathbf{m}}^{p}$ and $\psi_{\rho}^{p}$ that these functions on the hypergroup $X:=\Xi_{q} \times \underline{\mathrm{R}}$ are different for different indices.

We now prove that $\Sigma_{p, q}$ is equal to the complete dual space $\hat{X}$. For this consider the subgroup $G:=\{0\} \times \underline{\mathrm{R}}$ of $X$ as above and the associated annihilator

$$
A(\hat{X}, G):=\left\{\alpha \in \hat{X}:\left.\alpha\right|_{G} \equiv 1\right\} .
$$

This set is a closed subset of the dual $\hat{X}$ and can by [V2] be identified with the dual space of the quotient hypergroup $\left(\Xi_{q} \times \underline{\mathrm{R}}\right) / G$ which was studied in Remark 3.2(3). In fact, this hypergroup $\left(\Xi_{q} \times \underline{\mathrm{R}}\right) / G$ is (isomorphic to) a Bessel-type hypergroup on the Weyl chamber $\Xi_{q}$ as studied in section 4 of [R2]. We thus conclude readily from Section 4 of [R2] and the general results on annihilators in [V2] that $A(\hat{X}, G)=\Sigma_{p, q}^{2}$ holds, and that the mapping $E$ above restricted to $\Sigma_{p, q}^{2}$ is an homeomorphism.

We next turn to the complete dual $\hat{X}$. Here we first conclude from Theorem 3.15 and Corollary 3.5 that the dual $\hat{X}$ is the closure of $\Sigma_{p, q}^{1}$ in $C_{b}\left(\Xi_{q} \times \underline{\mathrm{R}}\right)$ w.r.t. the topology of locally uniform convergence. To prove that this closure is equal to $\Sigma_{p, q}$, we consider some $\alpha \in \hat{X} \subset C_{b}\left(\Xi_{q} \times \underline{\mathrm{R}}\right)$ which is the locally uniform limit of a sequence $\left(\varphi_{\lambda_{n}, \mathbf{m}_{\mathbf{n}}}^{p}\right)_{n \geq 1} \subset \Sigma_{p, q}^{1}$. As the restrictions to $G$ also converge locally uniformly, it follows from the definition of the $\varphi_{\lambda, \mathbf{m}}^{p}$ that $\left(\lambda_{n}\right)_{n \geq 1} \subset \underline{\mathrm{R}} \backslash\{0\}$ converges to some $\lambda \in \underline{\mathrm{R}}$. We consider two cases:

(1) If $\lambda=0$, then it follows from the definition of the $\varphi_{\lambda, \mathbf{m}}^{p}$ that $\alpha \in$ $A(\hat{X}, G)$, and the preceding consideration implies that $\alpha \in \Sigma_{p, q}^{2}$.

(2) Let $\lambda \neq 0$. If $\left(\mathbf{m}_{n}\right)_{n \geq 1}$ remains bounded, we may choose it as a constant sequence with $\mathbf{m}_{n}=\mathbf{m}$ without loss of generality, and we obtain from $\lambda_{n} \rightarrow \lambda$ that $\alpha=\lim _{n} \varphi_{\lambda_{n}, \mathbf{m}}^{p}=\varphi_{\lambda, \mathbf{m}}^{p} \in \Sigma_{p, q}^{1}$. We thus may restrict our attention to the case where at least one component of $\mathbf{m}_{n}$ tends to infinity. In this case we take an arbitrary multiindex $\mathbf{m}$ and conclude from the convergence of normalized Laguerre polynomials $L_{\lambda_{n}, \mathbf{m}}^{p}$ to 
$L_{\lambda, \mathbf{m}}^{p}$ (see Section 3.8) that for the density $h_{p, q}$ of (3.3)

$$
\begin{aligned}
\int_{\Xi_{q}} \alpha & (z, 0) \cdot \overline{\varphi_{\lambda, \mathbf{m}}^{p}(z, 0)} h_{p, q}(z) d z \\
\quad= & \int_{\Xi_{q}} \lim _{n \rightarrow \infty}\left(\varphi_{\lambda_{n}, \mathbf{m}_{n}}^{p}(z, 0) \cdot \overline{\varphi_{\lambda_{n}, \mathbf{m}}^{p}(z, 0)}\right) h_{p, q}(z) d z \\
\quad= & \int_{\Xi_{q}} \lim _{n \rightarrow \infty}\left(\frac{L_{\mathbf{m}_{n}}^{p}\left(\left|\lambda_{n}\right| z^{2} / 2\right) L_{\mathbf{m}}^{p}\left(\left|\lambda_{n}\right| z^{2} / 2\right)}{L_{\mathbf{m}_{n}}^{p}(0) L_{\mathbf{m}}^{p}(0)} \cdot e^{-\left|\lambda_{n}\right| \mid z \|^{2}}\right) h_{p, q}(z) d z .
\end{aligned}
$$

Using a renormalization as in Eq. (3.15) with $\lambda_{n} \rightarrow \lambda$, the dominated convergence theorem, and the fact that the modulus of the $\varphi_{\lambda_{n}, \mathbf{m}_{n}}^{p}$ is bounded by 1 , we obtain that that this expression is equal to

$$
\frac{1}{|\lambda|^{p q}} \lim _{n \rightarrow \infty} \int_{\Xi_{q}} \frac{L_{\mathbf{m}_{n}}^{p}\left(z^{2} / 2\right) L_{\mathbf{m}}^{p}\left(z^{2} / 2\right)}{L_{\mathbf{m}_{n}}^{p}(0) L_{\mathbf{m}}^{p}(0)} \cdot e^{-\|z\|^{2}} h_{p, q}(z) d z=0 .
$$

Therefore, as $\alpha \in C_{b}\left(\Xi_{q} \times\{0\}\right) \subset L^{2}\left(\Xi_{q}, e^{-\lambda\|z\|^{2}} h_{p, q}(z) d z\right)$, and as the Laguerre polynomials $\left(L_{\lambda, \mathbf{m}}^{p}\left(z^{2}\right)\right)_{\mathbf{m}}$ with squared arguments form an orthogonal basis of this $L^{2}$-space by Lemma 3.9, it follows that $\alpha=0$ a.s. on $\Xi_{q} \times\{0\}$ and thus on $\Xi_{q} \times \underline{\mathrm{R}}$. This is a contradiction of $\alpha$ being a continuous character with $\alpha(0,0)=1$.

Summarizing, we conclude that $\Sigma_{p, q}=\hat{X}$, and that a sequence $\left(\varphi_{\lambda_{n}, \mathbf{m}_{n}}^{p}\right)_{n \geq 1} \subset$ $\Sigma_{p, q}^{1}$ can converge to a character without loss of generality only in the following two cases: Either $\lambda_{n} \rightarrow \lambda \neq 0$ and $\left(\mathbf{m}_{n}\right)_{n}$ is finally constant or $\lambda_{n} \rightarrow 0$. In the first case, locally uniform convergence obviously appears, and in the second case we have locally uniform convergence for $\lambda_{n} \mathbf{m}_{n} \rightarrow \eta \in \Xi_{q}$ by Lemma 3.11.

We finally prove that a sequence $\left(\varphi_{\lambda_{n}, \mathbf{m}_{n}}^{p}\right)_{n \geq 1} \subset \Sigma_{p, q}^{1}$ can converge locally uniformly to some $\psi_{\rho}^{p}$ only for parameters with $\lim _{n} \lambda_{n} \mathbf{m}_{n}=\rho$. If this is done, it follows readily from our preceding informations that the mapping $E$ of our theorem is an homeomorphism. In order to prove this statement, consider such a sequence $\left(\varphi_{\lambda_{n}, \mathbf{m}_{n}}^{p}\right)_{n \geq 1}$ and its limit $\psi_{\rho}^{p}$. If the sequence $\left(\lambda_{n} \mathbf{m}_{n}\right)_{n} \subset \Xi_{q}$ is bounded, we find a convergent subsequence with some limit $\tilde{\rho} \in \Xi_{q}$. For this subsequence we obtain $\varphi_{\lambda_{n_{k}}, \mathbf{m}_{n_{k}}}^{p} \rightarrow \psi_{\tilde{\rho}}^{p}$ locally uniformly, and thus $\psi_{\tilde{\rho}}^{p}=\psi_{\rho}^{p}$, i.e., $\tilde{\rho}=\rho$. Therefore, each convergent subsequence of the bounded sequence $\left(\lambda_{n} \mathbf{m}_{n}\right)_{n} \subset \Xi_{q}$ converges to $\rho$ which implies $\lambda_{n} \mathbf{m}_{n} \rightarrow \rho$ as claimed. We finally consider the unbounded case. Here we may assume without loss of generality that the largest component of $\left(\lambda_{n} \mathbf{m}_{n}\right)_{n} \subset \Xi_{q}$ converges to $\infty$, i.e., that $\lambda_{n} \mathbf{m}_{1, n} \rightarrow \infty$. We then define $\tilde{\lambda}_{n}:=\rho / \mathbf{m}_{1, n}$ and observe that $\tilde{\lambda}_{n} \mathbf{m}_{n}$ has a subsequence which converges to some $\tilde{\rho} \in \Xi_{q}$ with $\tilde{\rho}_{1}=1$. For this subsequence and any $(\xi, t) \in \Xi_{q} \times \underline{\mathrm{R}}$ we the have

$$
\varphi_{\tilde{\lambda}_{n_{k}}, \mathbf{m}_{n_{k}}}^{p}(\xi, t) \rightarrow \psi_{\tilde{\rho}}^{p}(\xi, t)
$$


as well as by the definition of $\varphi_{\lambda, \mathbf{m}}^{p}$ and our assumption about locally uniform convergence,

$$
\varphi_{\tilde{\lambda}_{n_{k}}, \mathbf{m}_{n_{k}}}^{p}(\xi, t)=\varphi_{\lambda_{n_{k}}, \mathbf{m}_{n_{k}}}^{p}\left(\xi \cdot \sqrt{\tilde{\lambda}_{n_{k}} / \lambda_{n_{k}}}, t \cdot \tilde{\lambda}_{n_{k}} / \lambda_{n_{k}}\right) \rightarrow \psi_{\rho}^{p}(0,0)=1 .
$$

It follows that $\psi_{\tilde{\rho}}^{p} \equiv 1$ contradicting $\tilde{\rho}_{1}=1$. Therefore this limit case cannot appear which completes the proof of the theorem.

The proof of the continuity of the mapping $E$ above is quite special. It was pointed out to the author by J. Faraut that a more systematic approach for this part of the theorem is available by using heat kernels on the hypergroups $\Xi_{q} \times \underline{\mathrm{R}}$ and their explicit continuous hypergroup Fourier transfoms.

We finally turn to a refinement of the last part of the theorem. The following observation is clear from Section 2:

3.17 Lemma. Let $(\xi, a),(\eta, b) \in \Xi_{q} \times \underline{R}$ with $\xi_{q}>0$ and $\eta_{q}>0$. Then, for $p>2 q-1$, the convolution product $\delta_{(\xi, a)} \circ_{p, q} \delta_{(\eta, b)}$ is absolutely continuous w.r.t. the Haar measure $\tilde{\omega}_{p, q}$.

Proof. As absolute continuity is preserved under the continuous projection $\Phi: \Pi_{q} \times \underline{\mathrm{R}} \rightarrow \Xi_{q} \times \underline{\mathrm{R}}$, the lemma follows immediately from Remark 2.9.

As by the Riemann-Lebesgue Lemma for hypergroups (see [J]) the hypergroup Fourier transform maps functions in $L^{1}\left(\Xi_{q} \times \underline{\mathrm{R}}, \tilde{\omega}_{p, q}\right)$ to $C_{0}$-functions on the dual space $\hat{X}$, the preceding lemma and Theorem 3.16 imply the following.

3.18 Corollary. Let $(\xi, a) \in \Xi_{q} \times \underline{R}$ with $\xi_{q}>0$. Then, for $p>2 q-1$, the hypergroup Fourier transform of $\delta_{(\xi, a)}$ on the dual space $\hat{X}$ is a $C_{0}$-function. In particular, for $\xi \in \Xi_{q}$ with $\xi_{q}>0$,

$$
\lim _{\lambda \cdot m_{1} \rightarrow \infty} \frac{l_{\mathbf{m}}^{p}\left(\lambda \xi^{2} / 2\right)}{l_{\mathbf{m}}^{p}(0)}=0 .
$$

\section{A product formula for Laguerre functions}

In this section we derive a product formula for the multivariate Laguerre functions $l_{\mathbf{m}}^{p}$ of Section 3.8 for $p \geq 2 q-1$. For $q=1$, this formula was established directly by Koornwinder [Ko] who also discusses its connection with Heisenberg groups. We here derive the product formula from the product formula (3.2) for $p>2 q-1$ and its degenerate version for $p=2 q-1$ according to Remark $3.2(1)$ for the characters $\varphi_{\lambda, \mathbf{m}}^{p} \in \Sigma_{p, q}^{1}$ of the commutative hypergroups $\left(\Xi_{q} \times \underline{\mathrm{R}}, \circ_{p, q}\right)$. We here shall use the general approach of [RV] where, embedded into a more general setting, it is also explained how for $q=1$ Koornwinder's product formula for the one-dimensional Laguerre 
functions corresponds to the Heisenberg-type hypergroup convolution on $[0, \infty[\times \underline{\mathrm{R}}$. We now extend this approach from $q=1$ to $q \geq 1$.

For this we recapitulate from Remark 3.2 that $G:=\{0\} \times \underline{\mathrm{R}}$ is a subgroup of the commutative hypergroup $\left(\Xi_{q} \times \underline{\mathrm{R}}, \circ_{p, q}\right)$ for $p \geq 2 q-1$. Moreover, $\tau(x, t):=e^{i t}$ defines a function $\tau \in C_{b}\left(\Xi_{q} \times \underline{\mathrm{R}}\right)$ with

$$
|\tau(x, t)|=1, \quad \tau(\overline{(x, t)})=\overline{\tau(x, t)}, \quad \text { and } \quad \tau((x, t) \cdot(0, s))=\tau(x, t) \cdot \tau(0, s)
$$

for all $x \in \Xi_{q}$ and $s, t \in \underline{\mathrm{R}}$. In other words, $\tau$ is a partial character of $\left(\Xi_{q} \times \underline{\mathrm{R}}, \circ_{p, q}\right)$ with respect to $G$ according to Definition 4.1 of [RV]. We now consider the canonical projection

$$
p: \Xi_{q} \times \underline{\mathrm{R}} \rightarrow\left(\Xi_{q} \times \underline{\mathrm{R}}\right) / G \simeq \Xi_{q}
$$

according to Remark 3.2 (3) and recapitulate that the quotient hypergroup $\left(\Xi_{q} \times \underline{\mathrm{R}}\right) / G$ agrees with the corresponding Hermitian Bessel hypergroup on $\Xi_{q}$ of Rösler [R2], i.e., the corresponding hypergroup involution is the identity mapping. Following Section 4 of [RV], we now define a deformed quotient convolution of point measures on $\Xi_{q}$ by

$$
\delta_{\xi} \bullet_{\tau, p, q} \delta_{\eta}:=p\left(\tau \cdot\left(\delta_{(\xi, 0)} \circ_{p, q} \delta_{(\eta, 0)}\right)\right) \quad \text { for } \quad \xi, \eta \in \Xi_{q} .
$$

According to Section 4 of [RV], this convolution can be uniquely extended in a weakly continuous bilinear way to a commutative Banach-*-algebra $\left(M_{b}\left(\Xi_{q}\right), \bullet_{\tau, p, q}\right)$ with the total variation norm as norm. More precisely, by Theorem 4.6 and Corollary 4.7 of [RV], $\left(\Xi_{q}, \bullet_{\tau, p, q}\right)$ becomes a Hermitian signed hypergroup in the sense of [R1]; see also [RV] and [Ross] for the notion of signed hypergroups.

Let us compute the convolution (4.1) in an explicit way. Eq. (3.2) for $p>2 q-1$ shows that for $f \in C_{b}\left(\Xi_{q}\right)$ we have

$$
\begin{aligned}
& \delta_{\xi} \bullet_{\tau, p, q} \delta_{\eta}(f)=\int_{X i_{q}} f d p\left(\tau \cdot\left(\delta_{(\xi, 0)} \circ_{p, q} \delta_{(\eta, 0)}\right)\right) \\
& =\int_{\Xi_{q} \times \underline{\mathrm{R}}} f(x) \cdot e^{i t} d\left(\delta_{(\xi, 0)} \circ_{p, q} \delta_{(\eta, 0)}\right)(x, t) \\
& =\kappa_{p, q} \int_{B_{q}} \int_{U_{q}} f\left(\sigma\left(\sqrt{\xi^{2}+u \eta^{2} u^{*}+\xi w u \eta u^{*}+u \eta u^{*} w^{*} \xi}\right)\right) \\
& \cdot e^{-i \cdot \operatorname{Im} t r\left(\xi w u \eta u^{*}\right)} \cdot \Delta\left(I_{q}-w^{*} w\right)^{p-2 q} d u d w .
\end{aligned}
$$

For $p=2 q-1$ one obtains a corresponding degenerate version of this formula by using Remark 3.2(1) and Section 2.14. We notice that this convolution is obviously not probability preserving and usually even not positivity preserving.

Moreover it follows from Theorem 5.2 of [RV] that for $p \geq 2 q-1$ and all partitions $\mathbf{m}$, the normalized Laguerre functions

$$
\tilde{\varphi}_{\mathbf{m}}^{p}(x):=\frac{l_{\mathbf{m}}^{p}\left(x^{2} / 2\right)}{l_{\mathbf{m}}^{p}(0)}=e^{-\left(x_{1}^{2}+\ldots+x_{q}^{2}\right) / 2} \frac{L_{\mathbf{m}}^{p}\left(x^{2}\right)}{L_{\mathbf{m}}^{p}(0)} \quad\left(x \in \Xi_{q}\right)
$$


form all bounded $\underline{\mathrm{R}}$-valued multiplicative functions on $\left(\Xi_{q}, \bullet_{\tau, p, q}\right)$. In summary, we have the following product formula:

4.1 Corollary. For all $p>2 q-1, \xi, \eta \in \Xi_{q}$, and all partitions $\mathbf{m}$,

$$
\begin{gathered}
\tilde{\varphi}_{\mathbf{m}}^{p}(\xi) \cdot \tilde{\varphi}_{\mathbf{m}}^{p}(\eta)= \\
=\kappa_{p, q} \int_{B_{q}} \int_{U_{q}} \tilde{\varphi}_{\mathbf{m}}^{p}\left(\sigma\left(\sqrt{\xi^{2}+u \eta^{2} u^{*}+\xi w u \eta u^{*}+u \eta u^{*} w^{*} \xi}\right)\right) \\
\cdot e^{-i \cdot \operatorname{Im} t r\left(\xi w u \eta u^{*}\right)} \Delta\left(I_{q}-w^{*} w\right)^{p-2 q} d u d w .
\end{gathered}
$$

Moreover, for $p=2 q-1$,

$$
\begin{aligned}
& \tilde{\varphi}_{\mathbf{m}}^{2 q-1}(\xi) \cdot \tilde{\varphi}_{\mathbf{m}}^{2 q-1}(\eta)= \\
& =\kappa_{2 q-1, q} \int_{B^{q-1}} \int_{S} \int_{U_{q}} \tilde{\varphi}_{\mathbf{m}}^{2 q-1}\left(\sigma\left(\sqrt{\xi^{2}+\eta^{2}+\xi P(y) \eta+\xi P(y)^{*} \eta}\right)\right) \\
& \cdot e^{-i \cdot \operatorname{Im} t r\left(\xi P(y) u \eta u^{*}\right)} \cdot \prod_{j=1}^{q-1}\left(1-\left\|y_{j}\right\|_{2}^{2}\right)^{p-q-j} d u d y_{1} \ldots d y_{q-1} d s\left(y_{q}\right)
\end{aligned}
$$

with $y:=\left(y_{1}, \ldots, y_{q}\right)$ and $B:=\left\{y \in C^{q}:\|y\|_{2}<1\right\}$, where $s \in M^{1}(S)$ denotes the uniform distribution on the sphere $S:=\left\{y \in \underline{C}^{q}:\|y\|_{2}=1\right\}$, and $P$ is the map defined in (2.7).

As the convolution $\bullet_{\tau, p, q}$ of (4.2) is not mass preserving, there does not exist an associated translation invariant measure $m_{\tau, p, q} \in M^{+}\left(\Xi_{q}\right)$ in the usual hypergroup sense. However, Theorem 4.6 of [RV] ensures that the Haar measure

$$
d m_{p, q}(\xi)=\prod_{i=1}^{q} \xi_{i}^{2 p-2 q+1} \prod_{i<j}\left(\xi_{i}^{2}-\xi_{j}^{2}\right)^{2} d \xi \in M^{+}\left(\Xi_{q}\right)
$$

of the usual quotient hypergroup $\left(\Xi_{q} \times \underline{\mathrm{R}}\right) / G \simeq \Xi_{q}$ also admits the following adjoint relation for $\bullet_{\tau, p, q}$ : If for $f \in C_{c}\left(\Xi_{q}\right)$ and $\xi \in \Xi_{q}$ we define the translate

$$
T_{\xi} f(\eta):=\int_{\Xi_{q}} f d\left(\delta_{\xi} \bullet_{\tau, p, q} \delta_{\eta}\right),
$$

then we have for all $f, g \in C_{c}\left(\Xi_{q}\right)$

$$
\int_{\Xi_{q}}\left(T_{\xi} f\right) \cdot g d m_{p, q}=\int_{\Xi_{q}}\left(T_{\xi} g\right) \cdot f d m_{p, q} .
$$

4.2 Remark. Consider the group case with integer parameters $p \geq 2 q-1$. Here, all characters of the double coset hypergroup $\left(\Xi_{q} \times \underline{\mathrm{R}}, \circ_{p, q}\right)$ correspond to positive definite spherical functions on the Heisenberg group $H_{p, q}$ and admit therefore a dual product formula, i.e., for all $\alpha, \beta \in \Sigma_{p, q}$ (see Theorem 
3.16) there is a unique probability measure $\rho_{\alpha, \beta} \in M^{1}\left(\Sigma_{p, q}\right)$ such that for all $(x, t) \in \Xi_{q} \times \underline{\mathrm{R}}$,

$$
\alpha(x, t) \cdot \beta(x, t)=\int_{\Sigma_{p, q}} \gamma(x, t) d \rho_{\alpha, \beta}(\gamma)
$$

Let us take $\alpha(x, t):=e^{\lambda_{1} i t} \cdot \frac{l_{\mathbf{m}}^{p}\left(\left|\lambda_{1}\right| x^{2} / 2\right)}{l_{\mathbf{m}}^{p}(0)}$ and $\beta(x, t):=e^{\lambda_{2} i t} \cdot \frac{l_{\mathbf{n}}^{p}\left(\left|\lambda_{2}\right| x^{2} / 2\right)}{l_{\mathbf{n}}^{p}(0)}$ for $\lambda_{1}, \lambda_{2} \neq 0$ and partitions $\mathbf{m}, \mathbf{n}$.

If we consider the $t$-dependence of the product in (4.5) for $x=0$, we obtain for $\lambda_{1}, \lambda_{2}>0$ that

$$
\begin{aligned}
\frac{L_{\mathbf{m}}^{p}\left(\lambda_{1} x^{2} / 2\right)}{L_{\mathbf{m}}^{p}(0)} & \cdot \frac{L_{\mathbf{n}}^{p}\left(\lambda_{2} x^{2} / 2\right)}{L_{\mathbf{n}}^{p}(0)}= \\
& =\sum_{|\mathbf{k}| \leq|\mathbf{m}|+|\mathbf{n}|} c\left(\mathbf{m}, \mathbf{n}, \mathbf{k} ; \lambda_{1}, \lambda_{2}, p, q\right) \cdot \frac{L_{\mathbf{k}}^{p}\left(\left(\lambda_{1}+\lambda_{2}\right) x^{2} / 2\right)}{L_{\mathbf{k}}^{p}(0)}
\end{aligned}
$$

for unique coefficients $c\left(\mathbf{m}, \mathbf{n}, \mathbf{k} ; \lambda_{1}, \lambda_{2}, p, q\right)$, which satisfy

$$
c\left(\mathbf{m}, \mathbf{n}, \mathbf{k} ; \lambda_{1}, \lambda_{2}, p, q\right) \geq 0 \quad \text { and } \quad \sum_{\mathbf{k}} c\left(\mathbf{m}, \mathbf{n}, \mathbf{k} ; \lambda_{1}, \lambda_{2}, p, q\right)=1 .
$$

For instance, for $\mathbf{n}=\mathbf{0}$, we get for $0 \leq \lambda_{1} \leq \lambda_{2}$ that

$$
L_{\mathbf{m}}^{p}\left(\lambda_{1} x^{2} / 2\right)=\sum_{\mathbf{k}} c(\mathbf{m}, \mathbf{k} ; p, q) L_{\mathbf{k}}^{p}\left(\lambda_{2} x^{2} / 2\right)
$$

with nonnegative coefficients $c(\mathbf{m}, \mathbf{k} ; p, q)$.

On the other hand, for $\lambda_{1}>0$ and $-\lambda_{1}<\lambda_{2}<0$ we obtain

$$
\frac{l_{\mathbf{m}}^{p}\left(\lambda_{1} x^{2} / 2\right)}{l_{\mathbf{m}}^{p}(0)} \cdot \frac{l_{\mathbf{n}}^{p}\left(\left|\lambda_{2}\right| x^{2} / 2\right)}{l_{\mathbf{n}}^{p}(0)}=\sum_{\mathbf{k}} c\left(\mathbf{m}, \mathbf{n}, \mathbf{k} ; \lambda_{1}, \lambda_{2}, p, q\right) \cdot \frac{l_{\mathbf{k}}^{p}\left(\left(\lambda_{1}+\lambda_{2}\right) x^{2} / 2\right)}{l_{\mathbf{k}}^{p}(0)} .
$$

We expect that these and further related results also hold for arbitrary (noninteger) $p \geq 2 q-1$. For $q=1$, the formulas above are connected with the discrete Laguerre convolution in $[\mathrm{AG}$.

\section{References}

[AG] R. Askey, G. Gasper, Convolution structures for Laguerre polynomials. J. d'Anal. Math. 31 (1977), 233-248.

[BF] T.H. Baker, P.J. Forrester, The Calogero-Sutherland model and generalized classical polynomials. Comm. Math. Phys. 188 (1997), 175216.

[BJR1] C. Benson, J. Jenkins, G. Ratcliff, Bounded $K$-spherical functions on Heisenberg groups. J. Funct. Anal. 105 (1992), 409-443. 
[BJR2] C. Benson, J. Jenkins, G. Ratcliff, The spherical transform of a Schwartz function on the Heisenberg group. J. Funct. Anal. 154 (1998), 379-423.

[BJRW] C. Benson, J. Jenkins, G. Ratcliff, T. Worku, Spectra for Gelfand pairs associated with the Heisenberg group. Coll. Math. 71 (1996), 305-328.

[BH] W.R. Bloom, H. Heyer, Harmonic analysis of probability measures on hypergroups. De Gruyter Studies in Mathematics 20, de GruyterVerlag Berlin, New York 1995.

[C] G. Carcano, A commutativity condition for algebras of invariant functions. Boll. Un. Mat. Ital. 7 (1987), 1091-1105.

[dJ] M. de Jeu, Determinate multidimensional measures, the extended Carleman theorem and quasi-analytic weights. Ann. Probab. 31 (2003), 1205-1227.

[F] J. Faraut, Asymptotic spherical analysis on the Heisenberg group. Coll. Math. 118 (2010), 233-258.

[FK] J. Faraut, A. Korányi, Analysis on symmetric cones. Oxford Science Publications, Clarendon press, Oxford 1994.

[Ha] W. Hazod, Probability on matrix-cone hypergroups: limit theorems and structural properties. J. Appl. Anal. 15 (2009), 205-245.

[He] C.S. Herz, Bessel functions of matrix argument. Ann. Math. 61 (1955), 474-523.

[J] R.I. Jewett, Spaces with an abstract convolution of measures, Adv. Math. 18 (1975), 1-101.

[Kac] V. Kac, Some remarks on nilpotent orbits. J. of Algebra 64 (1980), 190-213.

[Kan] J. Kaneko, Selberg integrals and hypergeometric functions associated with Jack polynomials. SIAM J. Math. Anal. 24 (1993), 537-567.

[Ko] T. Koornwinder, The addition formula for Laguerre polynomials. SIAM J. Math. Anal. 8 (1977), 535-540.

[L] M. Lasalle. Une formule du binome généralisée pour les polynomes de Jack. C. R. Acad. Sci. 310 (1990), 253-256.

[M1] I.G. Macdonald, Some conjectures on root systems. SIAM J. Math. Anal. 13 (1982), 988-1007.

[M2] I.G. Macdonald, Symmetric functions and Hall polynomials. Oxford University Press 1995.

[R1] M. Rösler, Convolution algebras which are not necessarily probability preserving. In: Applications of hypergroups and related measure algebras (Summer Research Conference, Seattle, 1993). Contemp. Math. 183 (1995), 299 - 318.

[R2] M. Rösler, Bessel convolutions on matrix cones, Compos. Math. 143 (2007), 749-779. 
[R3] M. Rösler, Positive convolution structure for a class of HeckmanOpdam hypergeometric functions of type BC. J. Funct. Anal. 258 (2010), 2779-2800.

[RV] M. Rösler, M. Voit, Partial characters and signed quotient hypergroups. Can. J. Math. 51 (1999), 96-116.

[Ross] K. Ross. Signed hypergroups - a survey. In: Applications of hypergroups and related measure algebras (Summer Research Conference, Seattle, 1993) Contemp. Math. 183 (1995), 319 - 329.

[Ti] E.C. Titchmarsh, The theory of functions. Oxford Univ. Press, London, 1939.

[T] K. Trimeche, Generalized Wavelets and Hypergroups. Gordon and Breach, 1997.

[Vog] M. Vogel, Spectral synthesis on algebras of orthogonal polynomial series. Math. Z. 194 (1987), 99-116.

[V1] M. Voit, Positive characters on commutative hypergroups and some applications. Math. Z. 198 (1988), 405-421.

[V2] M. Voit, Duals of subhypergroups and quotients of commutative hypergroups. Math. Z. 210 (1992), 289-304.

[V3] M. Voit, Bessel convolutions on matrix cones: Algebraic properties and random walks. J. Theor. Probab. 22 (2009), $741-771$.

[W] J. A. Wolf, Harmonic analysis on commutative spaces. Amer. Math. Soc., 2007. 